\title{
A tissue-specific protein purification approach in Caenorhabditis elegans identifies novel interaction partners of DLG-1/Discs large
}

Selma Waaijers ${ }^{1,8}$, Javier Muñoz ${ }^{2,3,9}$, Christian Berends ${ }^{1}$, João J. Ramalho ${ }^{1}$, Soenita S. Goerdayal ${ }^{2,3}$, Teck Y. Low ${ }^{2,3}$, Adja D. Zoumaro-Djayoon ${ }^{2,3}$, Michael Hoffmann ${ }^{4}$, Thijs Koorman ${ }^{1,10}$, Roderick P. Tas ${ }^{5}$, Martin Harterink ${ }^{5}$, Stefanie Seelk ${ }^{6}$, Jana Kerver ${ }^{1}$, Casper C. Hoogenraad ${ }^{5}$, Olaf Bossinger ${ }^{7}$, Baris Tursun ${ }^{6 *}$, Sander van den Heuvel ${ }^{1}$, Albert J. R. Heck ${ }^{2,3}$ and Mike Boxem ${ }^{1 *}$ (D)

\begin{abstract}
Background: Affinity purification followed by mass spectrometry (AP/MS) is a widely used approach to identify protein interactions and complexes. In multicellular organisms, the accurate identification of protein complexes by AP/MS is complicated by the potential heterogeneity of complexes in different tissues. Here, we present an in vivo biotinylation-based approach for the tissue-specific purification of protein complexes from Caenorhabditis elegans. Tissue-specific biotinylation is achieved by the expression in select tissues of the bacterial biotin ligase BirA, which biotinylates proteins tagged with the Avi peptide.

Results: We generated N- and C-terminal tags combining GFP with the Avi peptide sequence, as well as four BirA driver lines expressing BirA ubiquitously and specifically in the seam and hyp7 epidermal cells, intestine, or neurons. We validated the ability of our approach to identify bona fide protein interactions by identifying the known LGL-1 interaction partners PAR-6 and PKC-3. Purification of the Discs large protein DLG-1 identified several candidate interaction partners, including the AAA-type ATPase ATAD-3 and the uncharacterized protein MAPH-1.1. We have identified the domains that mediate the DLG-1/ATAD-3 interaction, and show that this interaction contributes to C. elegans development. MAPH-1.1 co-purified specifically with DLG-1 purified from neurons, and shared limited homology with the microtubule-associated protein MAP1A, a known neuronal interaction partner of mammalian DLG4/PSD95. A CRISPR/Cas9-engineered GFP::MAPH-1.1 fusion was broadly expressed and co-localized with microtubules.
\end{abstract}

Conclusions: The method we present here is able to purify protein complexes from specific tissues. We uncovered a series of DLG-1 interactors, and conclude that ATAD-3 is a biologically relevant interaction partner of DLG-1. Finally, we conclude that MAPH-1.1 is a microtubule-associated protein of the MAP1 family and a candidate neuron-specific interaction partner of DLG-1.

Keywords: C. elegans, Mass spectrometry, Tissue-specific, Protein complex, Affinity purification, Microtubuleassociated, Discs large

\footnotetext{
* Correspondence: baris.tursun@mdc-berlin.de; m.boxem@uu.nl

${ }^{6}$ Berlin Institute for Medical Systems Biology (BIMSB), Max Delbrueck Center for Molecular Medicine (MDC) in the Helmholtz Association,

Robert-Roessle-Strasse 10, Berlin 13125, Germany

'Developmental Biology, Department of Biology, Faculty of Science, Utrecht

University, Padualaan 8, 3584 CH Utrecht, The Netherlands

Full list of author information is available at the end of the article
} 


\section{Background}

Physical interactions between proteins are essential for most, if not all, cellular processes. Hence, many smallscale studies focus on finding binding partners of individual proteins, and much effort has been put into identifying protein interactions on a large scale. One of the most commonly used techniques to identify protein complex components is affinity purification followed by mass spectrometry (AP/MS). Insight into protein complex composition obtained by AP/MS has already had a major impact on our understanding of cellular processes and signal transduction pathways [1]. Systematic AP/MS efforts have mostly focused on single-cell systems, such as yeast, bacteria, and cultured Drosophila melanogaster cells [2-8]. In multicellular organisms, the composition and function of protein complexes containing a particular protein of interest may differ between cell types or tissues [9]. Purifying a protein from whole-animal lysates will result in the identification of members of all complexes, which complicates the interpretation of the biological meaning of the identified interactors. One approach to overcome this limitation is to purify specific tissues or cell types. However, the purification of specific cell types from model organisms is challenging at the scale needed for AP/MS.

Here, we present a method for the tissue-specific purification of protein complexes from the nematode Caenorhabditis elegans, based on cell type-specific biotinylation. C. elegans is a widely used multicellular model organism that contains many differentiated cell types and tissues, including epithelia, neurons, and muscle. Several studies have used AP/MS approaches to purify $C$. elegans proteins and identify interaction partners [10-22]. However, tissue-specific AP/MS approaches are not widely used.

To purify proteins from specific C. elegans tissues, we adapted a system based on in vivo biotinylation of a protein of interest. In this approach, a protein of interest is tagged with the 15-amino acid Avi-tag, which can be biotinylated in vivo by the BirA biotin ligase from Escherichia coli $[23,24]$. The biotinylated bait protein and any associated proteins are then purified with streptavidincoated beads, and their identities are determined by mass spectrometry (MS). We demonstrate the applicability of this approach by showing tissue-specific biotinylation of Avi-tagged GFP, and by identifying the wellknown LGL-1/PAR-6/PKC-3 complex in two epithelial tissues. We also identify a novel ubiquitous interaction between the $C$. elegans Discs large protein DLG-1, and the mitochondrial AAA-type ATPase ATAD-3. We confirm this surprising interaction by immunoprecipitation, map the ATAD-3 interaction domain by yeast twohybrid assays, and confirm that this domain mediates the interaction in vivo. Finally, we identify a candidate neuron-specific interaction between DLG-1 and MAPH1.1, an uncharacterized protein that shares some sequence similarity with mammalian microtubuleassociated proteins MAP1A, MAP1B, and MAP1S. We show that MAPH-1.1 is a microtubule-associated protein, and likely represents a $C$. elegans MAP1 family member.

\section{Results}

\section{A biotinylation-based tissue-specific protein purification} approach

To purify protein from specific tissues, we express Avitagged proteins of interest from their native regulatory sequences, while biotinylation in a specific tissue is accomplished by expressing BirA from tissue-specific promoters (Fig. 1a). Expression of the bait protein from its native regulatory sequences has several advantages. First, expression will closely mimic the endogenous expression pattern. Second, functionality of the tagged protein can be tested by crossing the transgenic strain with a strain carrying a mutation in the corresponding gene. This simultaneously creates a strain that does not express the untagged endogenous protein. Finally, only a single transgenic strain needs to be created, which can then be crossed to multiple BirA driver strains to purify the protein of interest from different tissues (Fig. 1a). As a first step, we generated four distinct BirA driver strains expressing C. elegans codon-optimized and Myc-tagged BirA ligase under the control of different promoters. Two transgenic strains express BirA in epithelial tissues, one expresses BirA in the intestine from the elt-2 promoter, and one expresses BirA in the seam and hyp7 epidermal cells from the wrt-2 promoter (wrt-2 expresses predominantly in seam cells, with weak expression in hyp7) [25]. In addition, we generated strains expressing BirA ubiquitously (using the rps-27 promoter), and in neuronal cells (using the rgef- 1 promoter). Reverse transcription (RT)-PCR of transgenic animals expressing BirA from the rgef-1 promoter showed that the BirA transgene was properly transcribed and spliced (Additional file 1: Figure S1).

We designed $\mathrm{N}$ - and $\mathrm{C}$-terminal tags consisting of GFP and the Avi sequence, separated by two Tobacco etch virus (TEV) cleavage sites, which we term the GTA tag (for GFP, TEV, Avi) (Fig. 1b, Additional file 2: Figure S2, and Additional file 3: Figure S3). The presence of GFP enables the examination of the expression pattern and subcellular localization of the protein of interest, either to confirm that the tagged protein localizes as expected or to study the localization of proteins whose localization pattern has not yet been (fully) described. Tags combining GFP with affinity purification, including a similar tag incorporating the Avi sequence, 


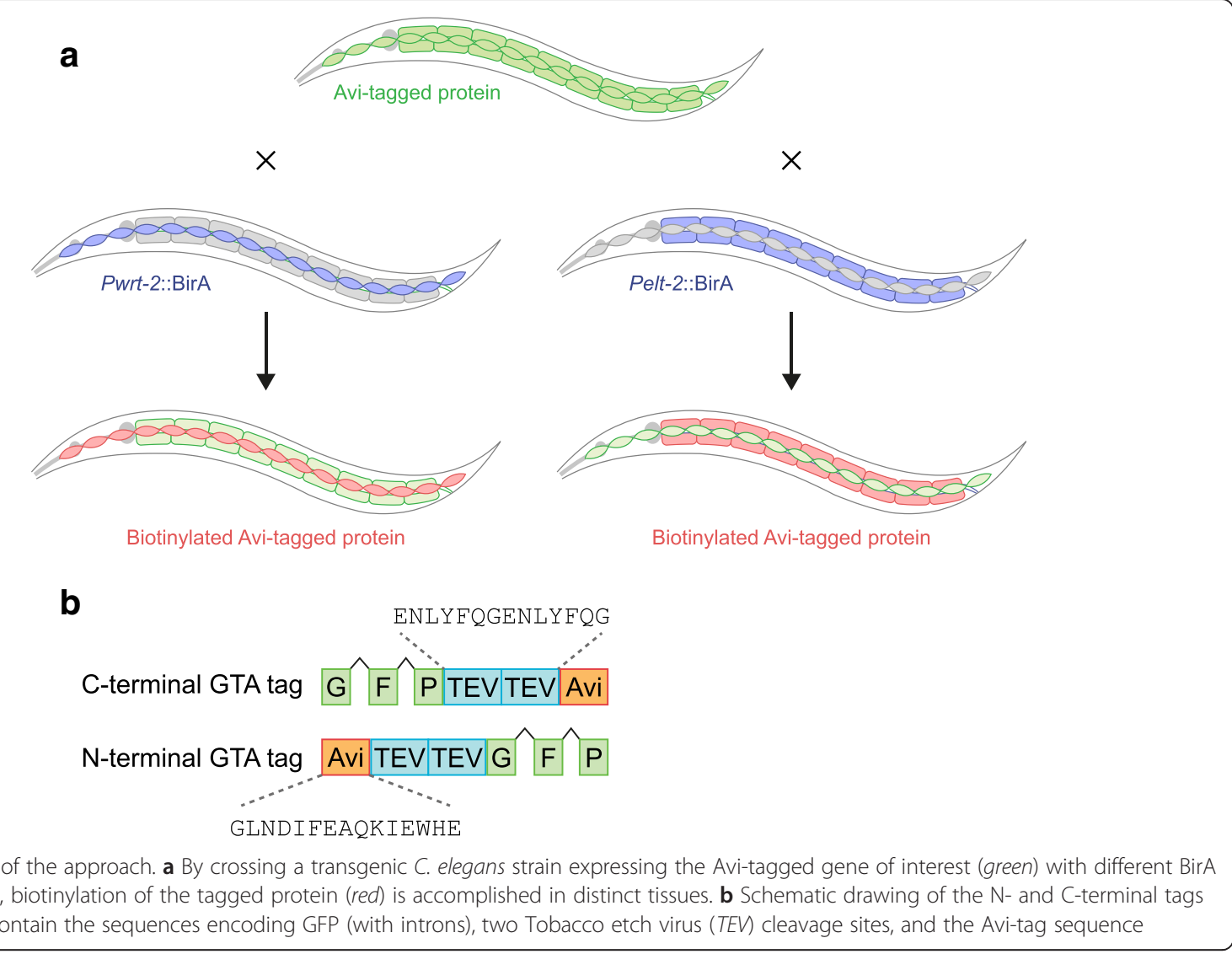

have been used successfully in C. elegans $[10,19]$. The use of TEV cleavage sites is necessary to eliminate biotinylated proteins naturally present in C. elegans, which will also bind to streptavidin-coated beads. TEV cleavage releases the bait protein and any associated proteins from the beads, while naturally biotinylated background proteins remain bound. GFP, TEV, and Avi are separated by short flexible linkers of five small amino acids, while GFP is separated from the bait protein by a longer flexible linker of 13 small amino acids (Additional file 1: Figure S1 and Additional file 2: Figure S2).

To add the tags to genes encoding proteins of interest, we used fosmid recombineering, a homologous recombination-based genetic engineering technique in bacteria [26]. This approach enabled us to integrate the tag into large regions of genomic DNA $(30-40 \mathrm{~kb})$ that likely contain all the native regulatory sequences of the gene of interest, including the promoter, $3^{\prime}$ UTR, and introns (Fig. 1b).

An overview of the entire tagging and AP/MS procedure is shown in Fig. 2. Transgenic strains expressing GTA-tagged proteins are generated by germline injection of engineered fosmids, and the transgenic array is integrated into the genome by gamma irradiation.
Transgenic strains expressing the GTA-tagged bait protein are then crossed to BirA-expressing strains. Whenever possible, transgenic strains are also crossed to strains carrying a mutation in the endogenous protein of interest. This allows testing to determine if the tagged protein is fully functional and eliminates the presence of wild-type untagged protein, which would otherwise reduce the fraction of complexes incorporating a tagged protein. The strains are grown at a large scale in liquid culture before harvesting, lysis, and purification of biotinylated proteins with streptavidin-coated beads. The bait protein and any bound proteins are then cleaved off the beads using TEV protease, and analyzed by tandem mass spectrometry (MS/MS) to determine their identities.

\section{In vivo biotinylation and purification are highly tissue- specific}

Tissue-specific expression of BirA should result in purification of the bait protein specifically from that tissue. However, at least two potential problems might give rise to biotinylation - and thus purification - of proteins from unintended tissues. First, if the expression of BirA is not tightly limited to the tissue of interest, BirA might be expressed at low levels in other tissues. However, 


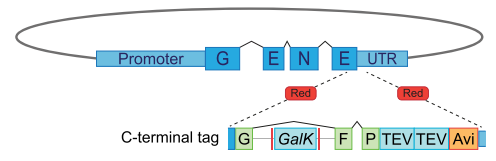

a. Add GTA tag to gene of interest by fosmid-based recombineering

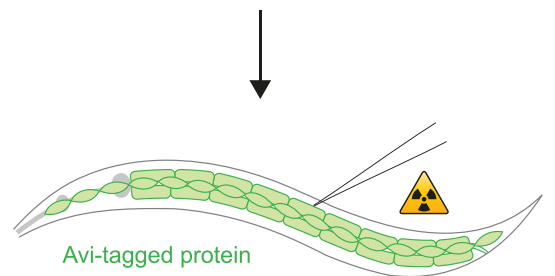

b. Generate integrated transgenic line by microinjection and g-irradiation

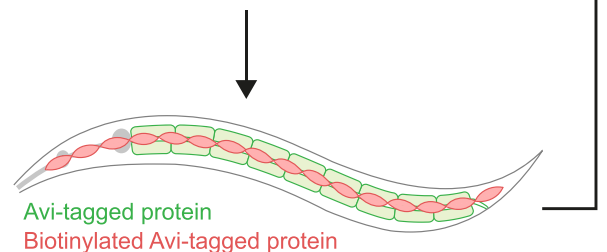

C. Cross with BirA strains, and if possible with mutants lacking endogenous protein

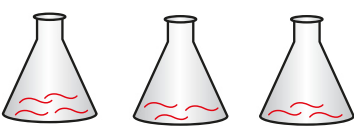

d. Grow in triplicate in liquid culture

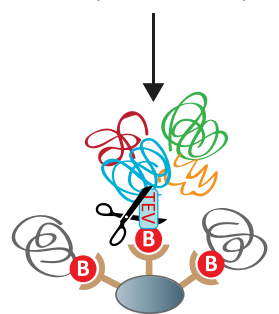

e. Purify bait with streptavidin beads, release from beads with TEV cleavage

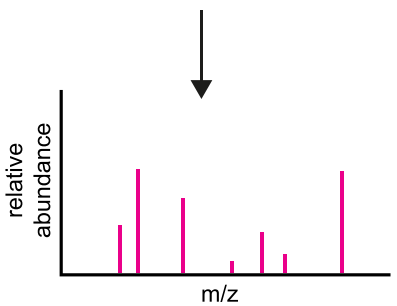

f. Identify proteins by MS/MS

Fig. $\mathbf{2}$ Schematic of the workflow. a The GTA tag is added to a gene of interest using recombineering. $\mathbf{b}$ Transgenic C. elegans strains expressing the GTA-tagged protein are generated by injection followed by gamma irradiation-mediated integration of the extrachromosomal array. $\mathbf{c}$ Transgenic strains are crossed with strains expressing BirA from a tissue-specific promoter, and with a genetic null mutant if appropriate. d The transgenic strains are grown in triplicate in liquid culture. e Affinity purification is performed on whole-animal lysates. The bait protein with any interacting proteins is subsequently cleaved off the beads by Tobacco etch virus (TEV) protease. $\mathbf{f}$ The samples are analyzed by tandem mass spectrometry (MS/MS) to identify the proteins they contain

many promoters with well-documented and highly tissue-specific expression patterns have been identified in C. elegans. Second, BirA might biotinylate Avi-tagged proteins after the lysis procedure, when proteins from the entire animal are mixed together. The risk of this is low, as BirA activity requires the presence of bivalent ions, which are chelated by the EDTA in the lysis buffer [27]. Nevertheless, we first wished to demonstrate the specificity of biotinylation and purification from a specific tissue.

To test the tissue specificity of our approach, we generated transgenic $C$. elegans strains expressing cytoplasmic Avi-tagged GFP from the intestinal elt-2 promoter and the seam and hyp7 epidermal wrt-2 promoter. Both GTA strains showed expression of cytoplasmic GFP in the expected cells (Fig. 3a, b). We crossed each GTA strain with both epithelial BirA driver strains. Of the four resulting strains, two expressed the biotin ligase and GTA in the same tissue (intestine or epidermal cells), while the other two expressed BirA and GTA in two different tissues. Animals of all four strains were lysed, biotinylated proteins were purified using streptavidin-coated beads, and the presence of GFP was examined by western blot with an antibody directed against GFP. All samples showed the expected purification pattern: when BirA and GTA were present in the same tissue, GTA was purified by the streptavidin beads; when BirA and GTA were expressed in separate tissues, no GTA was purified (Fig. 3c). Thus, in vivo biotinylation and protein purification are highly tissue specific.

\section{GTA-tagged bait proteins are functional and recapitulate known localization patterns}

To test the tissue-specific purification approach, we tagged five polarity regulators that show distinct subcellular localization patterns with the GTA tag: the apical protein PAR-3, the junctional protein DLG-1, the basolateral proteins LET-413 and LGL-1, and the cortical protein CDC-42. These five proteins all contain multiple protein-protein interaction domains and for some of these proteins, interacting partners have already been identified in C. elegans or other systems. For example, DLG-1 interacts with the C. elegans-specific protein AJM-1, whereas PAR-3 and LGL-1 can both form a 


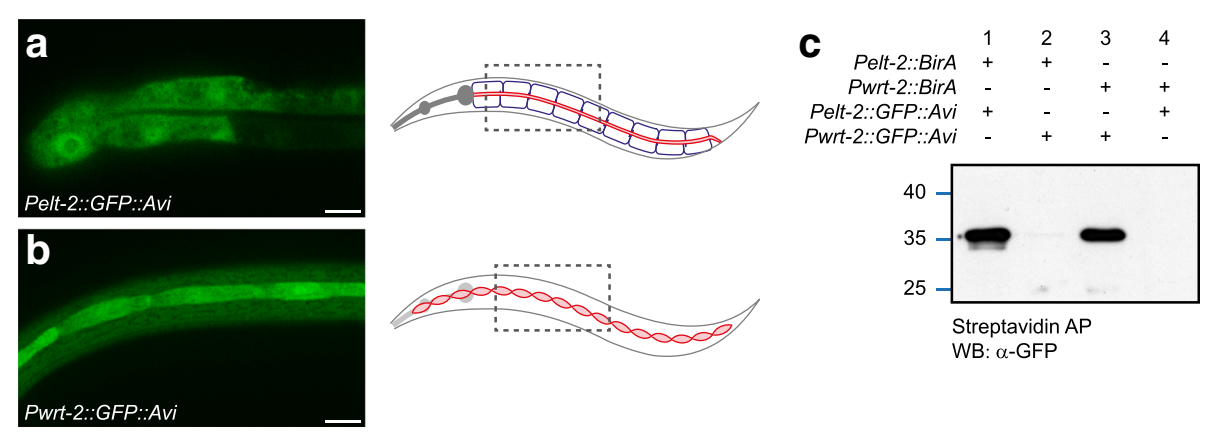

Fig. 3 In vivo biotinylation is highly tissue-specific. a Expression of GFP::TEV::Avi from the intestinal elt-2 promoter. $\mathbf{b}$ Expression of GFP::TEV::Avi from the seam and hyp7 epidermal cell-specific wrt-2 promoter. In a and b, the tissue and approximate region imaged are indicated in the cartoon worm. c Western blot detection of GFP::TEV::Avi purified with streptavidin beads from lysates obtained from animals expressing GFP::TEV::Avi and BirA in the same tissue (lanes 1 and 3) or in different tissues (lanes 2 and 4). Scale bars are $10 \mu \mathrm{m}$

complex with PKC-3 and PAR-6, and CDC-42 binds to PAR-6 [28-33]. For PAR-3, DLG-1, LET-413, and LGL-1, we added the GTA tag to the C-terminus, as C-terminal tags are more often compatible with protein function than N-terminal tags [34]. For CDC-42, we used the $\mathrm{N}$-terminal tag, as it has already been shown that $C$. elegans GFP::CDC-42 is able to localize to the cortex, similar to wild-type CDC-42 [28]. For all five genes, we successfully generated integrated transgenic strains using the procedure outlined in Fig. 2. We examined the expression pattern of the tagged bait proteins in the intestine and seam cells and found that all proteins localized to the expected subcellular domains (Fig. 4). In both tissues we observed junctional localization of DLG-1::GTA, basolateral localization of LET-413::GTA and LGL-1::GTA, and cortical localization of GTA::CDC-42. PAR3::GTA was visible at the apical surface of the seam cells. Unexpectedly, no tagged PAR-3 was detected in the intestinal epithelium. In addition to the epithelial localization, we also observed expression of DLG-1::GTA in ventral cord neurons (Fig. 4d), which is consistent with the expression in neurons of the human homolog DLG4 (PSD-95) [35].

We crossed the $d l g-1, l g l-1$, and let-413 GTA-tagged transgenic strains with strains carrying a mutant allele of the corresponding endogenous gene. $c d c-42$ and par-3 null alleles are early embryonic lethal $[36,37]$. Transgenes are usually silenced in the germline and are therefore unlikely to rescue these phenotypes. Consequently, we did not use mutant backgrounds for these two bait proteins.

The $d \lg -1:: G T A$ transgenic strain was crossed with a strain carrying the $d l g-1$ (ok318) deletion allele, which causes embryonic arrest at the 2-fold stage. Homozygous $d l g$-1(ok318) mutants carrying the $d l g-1:: G T A$ transgene were fully viable, demonstrating that the DLG-1::GTA protein is functional. Loss of $l g l-1$ does not affect cell polarity or viability, preventing a complementation test of the functionality of the $\operatorname{lgl} 1:: G T A$ transgene $[38,39]$. Nevertheless, we crossed our $l g l-1:: G T A$ transgenic strain with the $l g l-1(t m 2616)$ deletion allele to prevent incorporation of untagged LGL-1 protein into complexes. For let-413, no molecular null allele is available, and the let-413::GTA transgenic strain was crossed with a strain carrying the let413(s128) missense mutation, which causes lethality during elongation of the developing embryo [40]. While expression of LET-413::GTA restored embryonic viability, this strain still grew slowly. LET-413 purifications where therefore performed in a let-413 wild-type background.

In summary, the five tagged proteins localize to the appropriate subcellular domains, and the two transgenes that we could test in complementation assays rescue the lethality caused by the corresponding mutant alleles. Thus, the experimental design appears well suited for the tagging of proteins of interest, without interfering with their function.

\section{Large-scale culturing and purification of protein complexes}

Each of the five transgenic strains were crossed with the Prps-27::BirA (ubiquitous), Pelt-2::BirA (intestine), and Pwrt-2::BirA (epidermal) BirA driver strains. Because of the observed neuronal localization of DLG-1, the $d l g$ $1:: G T A$ strain was also crossed with the pan-neuronal Prgef-1::BirA transgenic strain (Table 1). As described above, the $d l g-1:: G T A$ and $l g l-1:: G T A$ transgenic strains also carried a putative null mutation in the corresponding endogenous gene. As a negative control to identify common contaminant proteins or proteins that bind to the GTA tag, we generated a transgenic strain expressing cytoplasmic GFP::TEV::Avi from the ubiquitous rps-27 promoter, which was crossed with each of the four BirA driver strains. In total 20 transgenic strains were generated for affinity purification: strains expressing one of the five tagged bait proteins or the GFP::TEV::Avi control construct, combined with BirA expression from one 


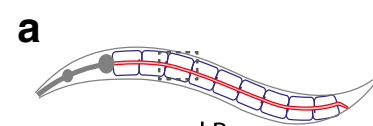

panel B

b
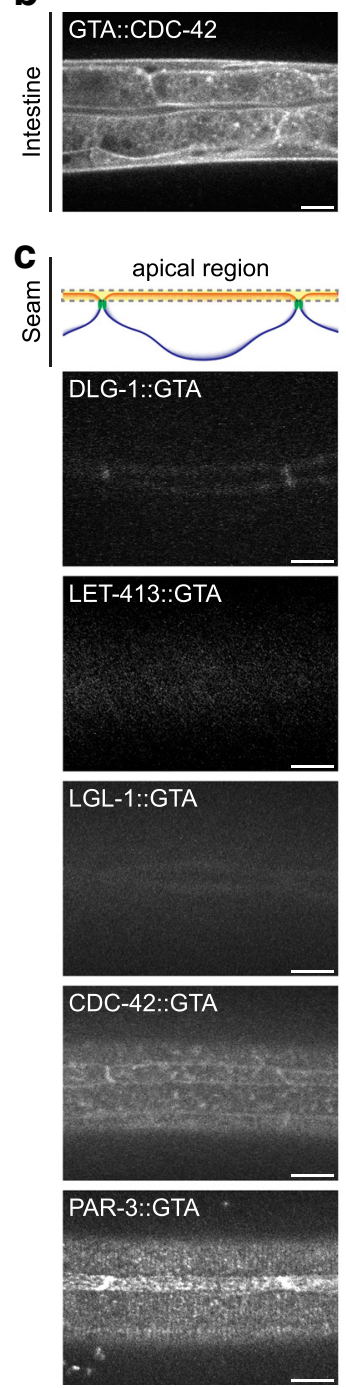

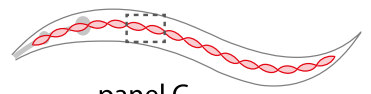

panel C
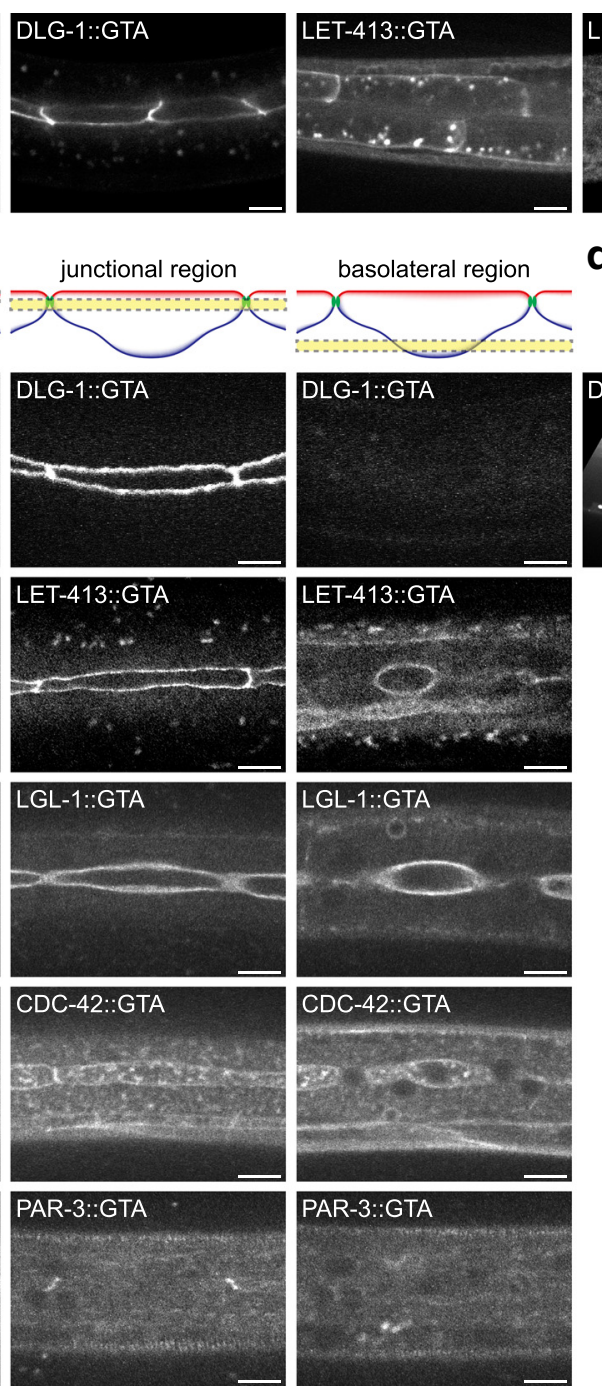

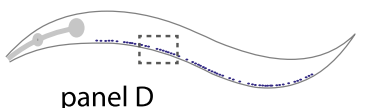

panel D

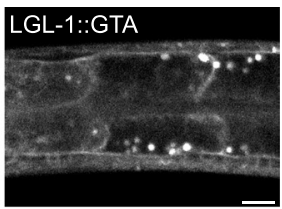

d

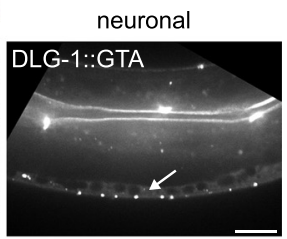

Fig. 4 Spinning disc confocal immunofluorescence microscopy images of subcellular localization patterns of GTA-tagged bait proteins. a Schematic representation of the areas imaged. b Localization in intestinal cells. c Localization in seam cells. In both tissues, CDC-42 localizes to cortex, DLG-1 localizes to cell junctions, and LET-413 and LGL-1 localize to basolateral cortex. PAR-3 localizes apically in seam cells, while no PAR-3 expression was detected in intestine. d Expression of DLG-1:GTA in ventral cord neurons (e.g., white arrow). Scale bars are $10 \mu \mathrm{m}$

of three different promoters, and in addition, two strains expressing dlg-1::GTA or GFP::TEV::Avi transgenes combined with BirA expression under the control of the neuronal rgef-1 promoter.

Each of the 20 strains was grown in large-scale liquid culture, starting from semi-synchronized L1 cultures generated by starvation on plates. After growth in liquid culture, animals were harvested when most larvae were at the third or fourth larval stage. All strains were grown and analyzed in triplicate to enhance the possibility of separating bona fide protein-protein interactions from nonspecific interactions. All of the harvested cultures were lysed by sonication, and biotinylated proteins were purified using streptavidin-coated beads. Next, bait proteins and binding partners were released from the streptavidin beads through TEV protease cleavage.

To examine the specificity of the biotinylation by BirA, and the efficiency of the purification and subsequent release by TEV cleavage, we analyzed expression and purification of DLG-1::GTA (Fig. 5). Western blot analysis of 
Table 1 Lines generated for purification

\begin{tabular}{llll}
\hline BirA driver & GTA-tagged bait & BirA driver & GTA-tagged bait \\
\hline Prps-27::BirA & par-3::GTA & Pwrt-2::BirA & par-3::GTA \\
& dlg-1::GTA & & dlg-1::GTA \\
& let-413::GTA & & let-413::GTA \\
& lgl-1::GTA & & lgl-1::GTA \\
& GTA::cdc-42 & & GTA::cdc-42 \\
Pelt-2::BirA & par-3::GTA & Prgef-1::BirA & dlg-1::GTA \\
& dlg-1::GTA & & \\
& let-413::GTA & & \\
& lgl-1::GTA & & \\
& GTA::cdc-42 & & \\
\hline
\end{tabular}

biotinylated proteins in whole lysates showed a band of the correct molecular weight in samples expressing DLG-1::GTA and ubiquitous BirA, which was absent from the wild-type N2 control (Fig. 5a). No additional biotinylated bands were detected upon expression of BirA, indicating that BirA specifically biotinylates the Avi-tag. Analysis of beads and eluate after purification with streptavidin beads and TEV cleavage showed that DLG-1::GFP was released from the beads, while endogenously biotinylated proteins remained bound (Fig. 5b). We also analyzed the biotinylation efficiency and recovery by
TEV cleavage of GTA::CDC-42. We purified GTA::CDC42 from a strain expressing BirA in the seam and hyp7 epidermal cells using GFP-Trap beads as well as streptavidin beads (Fig. 5c). The equal levels of recovery (compare lanes 1 and 2) indicate efficient biotinylation of GTA::CDC-42. Analysis of beads and eluate after TEV cleavage of GTA::CDC-42 purified with streptavidin beads indicate efficient TEV cleavage. However, approximately half of the CDC-42 remained bound to the beads, despite having been cleaved by TEV (as indicated by the shift in apparent molecular weight). Thus, the overall efficiency of recovery can vary between bait proteins.

\section{Tissue-specific protein purification recovers known LGL-1 interactors}

We next analyzed the samples by high-resolution MS. Each sample was run on a sodium dodecyl sulfate polyacrylamide gel electrophoresis (SDS-PAGE) gel for a length of $\sim 1 \mathrm{~cm}$, followed by in-gel digestion with trypsin and liquid chromatography (LC) MS/MS analysis. Raw data are available via ProteomeXchange with identifier PXD002139. To distinguish genuine interacting proteins from nonspecific binding proteins, we analyzed the data using the SAINT

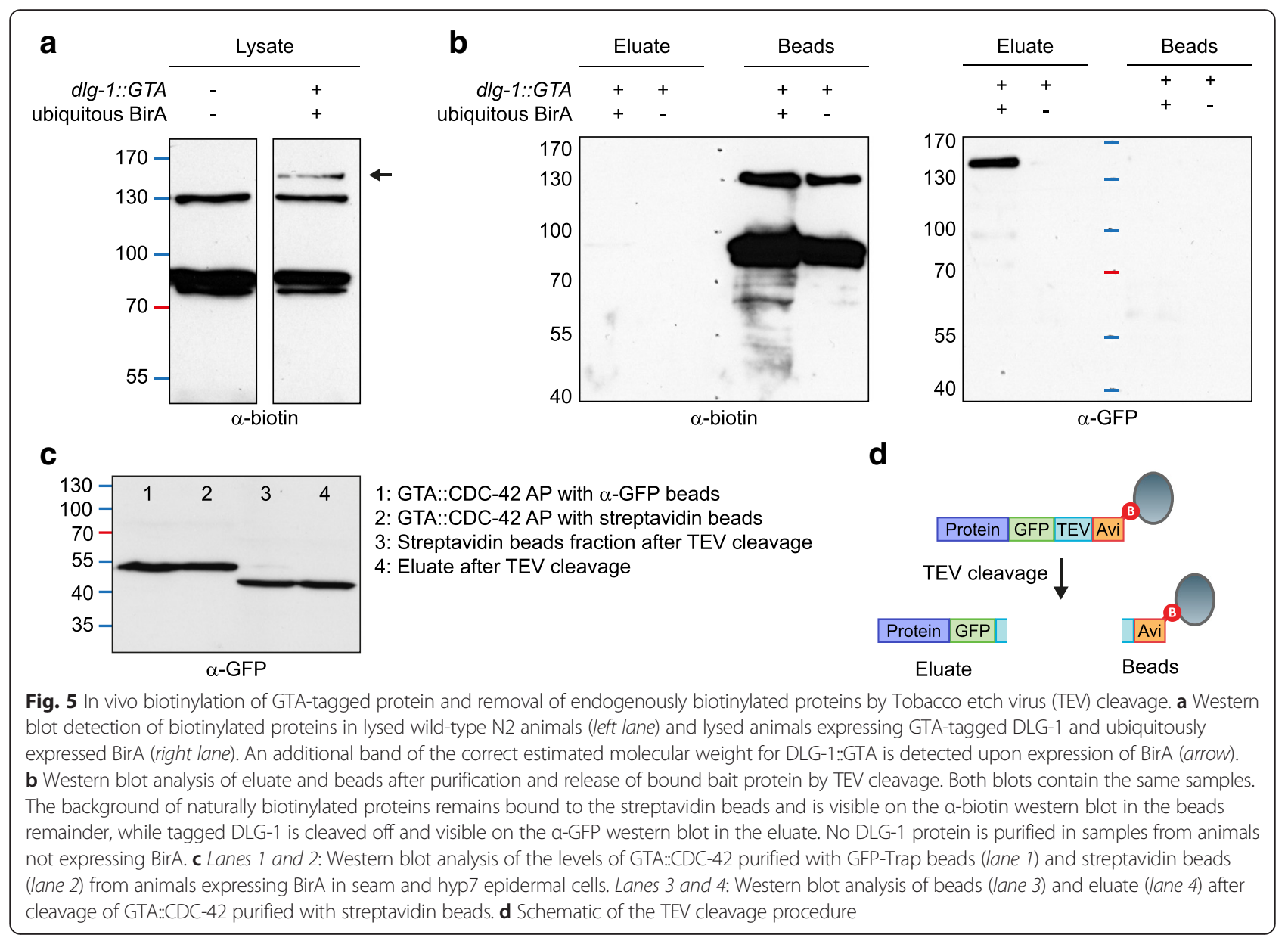


tool, which was developed to assign confidence scores to protein-protein interactions identified in AP/MS experiments [41]. Based on label-free quantification data, and taking into account the number of replicates in which a protein is observed, as well as negative control data, SAINT assigns a probability score to each bait-prey pair identified [41]. Scores range from 0 to 1 , and interactions scoring $>0.8$ can be considered to represent high confidence interactions [41]. We analyzed our samples using SAINT Express embedded within the CRAPome online interface [42]. Spectral counts for the triplicate experiments and negative control samples were uploaded and processed using the default settings. The four tissues analyzed (ubiquitous, epidermal, intestine, neuronal) were processed separately. The results are presented in Additional file 4: Table S1 and Additional file 5: Table S2.

The bait proteins LGL-1, CDC-42, DLG-1, and LET413 were each themselves identified with high confidence: SAINT probability scores were $\geq 0.98$ in all tissues examined, with the exception of LET-413 in the intestine (score of 0.86). Bait protein PAR-3 was only identified in the ubiquitous sample, with a SAINT score of 0.65. Because PAR-3::GTA was readily detected by microscopy, the protein is likely not efficiently recovered in our purification procedure. PAR-3 samples were therefore excluded from further analysis. The remaining four baits together identified 55 high confidence interactions, which were identified in at least one tissue with a SAINT score $>0.8$. However, 24 of these were ribosomal proteins or proteins associated with ribosome biogenesis, and most of these were identified with multiple bait proteins (22/24 were found by all four baits). Disregarding interactions with these ribosome-associated proteins, we identified 25 interactions with DLG-1, two interactions with LET-413, and three interactions with LGL-1 (Table 2 and Additional file 6: Table S3). No interactions with a SAINT score $>0.8$ were identified with CDC-42. However, in an independent experiment, CDC-42 co-purified with the RhoGDI RHI-1, a known interactor of CDC-42 [43] (not shown), which indicates that adjusting purification conditions may yield additional interacting proteins.

The proteins that co-purified with LET-413 appear unlikely to be specifically involved in cell polarity: B0303.3 is a homolog of $\beta$-ketothiolase, and QARS- 1 is a glutaminyl tRNA synthetase. In contrast, LGL-1 co-purified with PAR-6 and PKC-3. C. elegans LGL-1 was previously found to be associated with PAR-6 and PKC-3 in embryos [39], and Drosophila and mammalian Lgl form a complex with Par-6 and aPKC [29, 32, 33]. In our experiments, PAR- 6 and PKC-3 both co-purified with LGL-1 biotinylated specifically in the intestine or seam and hyp7 epidermal cells, as well as with ubiquitously biotinylated LGL-1. These results demonstrate that our biotinylation-based approach is able to identify biologically relevant interactions from specific tissues with high confidence.

\section{Identification of a novel interaction between DLG-1 and the AAA ATPase-family protein ATAD-3}

To validate the ability of our approach to identify novel interactors, we focused on the candidate DLG-1 interaction partner ATAD-3, which was identified with high confidence using ubiquitously biotinylated DLG-1::GTA, as well as with lower confidence using BirA expressed in epidermal cells, intestine, and neurons (Table 2). Moreover, ATAD-3 was not identified with any confidence with the other bait proteins (Additional file 5: Table S2). ATAD-3 is an evolutionarily conserved AAA-family ATPase that is essential for mitochondrial activity and development of C. elegans [44]. We verified the ubiquitous nature of the DLG-1/ATAD-3 interaction by immunoprecipitating ATAD-3 and DLG-1 from embryonic lysates using specific antibodies. Purification of ATAD-3 revealed co-immunoprecipitation of DLG1 at a level that was comparable to that observed after DLG-1 immunoprecipitation (Fig. 6a, compare lane 7 and 4). This suggests that a major fraction of DLG-1 interacts with ATAD-3 in the embryo. Conversely, purification of DLG-1 resulted in the co-immunoprecipitation of only a small amount of ATAD-3 (Fig. 6a, lane 4). Purification of ATAD-3 or DLG-1 from lysates of embryos treated with RNAi directed against $d l g-1$ or atad-3 failed to coimmunoprecipitate substantial amounts of DLG-1 or ATAD-3. Thus, the co-immunoprecipitation of DLG-1 is dependent on the presence of ATAD-3, and vice versa. Together, these data indicate that DLG-1 specifically interacts with ATAD-3.

DLG-1 contains three PDZ domains, which are known to function as protein interaction modules. Yeast two-hybrid experiments with fragments of DLG-1 revealed that ATAD-3 binds to the second PDZ domain of DLG-1 (Fig. 6b). PDZ domains frequently bind to short C-terminal motifs in interacting proteins, and the final four amino acids of ATAD-3 (ETAV) match a predicted PDZ domainbinding-specificity class [45]. Indeed, removal of the ETAV sequence rendered ATAD-3 incapable of binding to DLG-1 in the yeast two-hybrid system (Fig. 6b). This indicates that ATAD-3 binds with its C-terminal ETAV motif to the second PDZ domain of DLG-1. To investigate whether the ETAV motif is necessary in vivo to mediate the interaction of ATAD-3 with DLG-1, we generated strains expressing full-length ATAD-3 (ATAD- $3^{\mathrm{FL}}$ ), or ATAD-3 lacking the C-terminal four amino acids (ATAD ${ }^{\triangle \mathrm{ETAV}}$ ). We inserted single-copy transgenes in chromosome IV by MosSCI [46], and crossed these animals with the atad-3(ok3093) strain to obtain animals expressing only the transgene. Both strains express ATAD-3 at levels comparable to wild type (Fig. 6c and Additional file 7: Figure S4). Co-immunoprecipitation experiments showed that $\mathrm{ATAD}^{\mathrm{FL}}$ interacts with DLG-1 in 
Table 2 Candidate interactors identified

\begin{tabular}{|c|c|c|c|c|c|c|}
\hline \multirow[t]{2}{*}{ Bait } & \multirow[t]{2}{*}{ Interactor } & \multicolumn{4}{|c|}{ SAINT confidence scores } & \multirow[t]{2}{*}{ Description } \\
\hline & & Ubiquitous & Intestine & Seam/hyp7 & Neuron & \\
\hline CDC-42 & $\mathrm{CDC}-42$ & 1.00 & 1.00 & 1.00 & - & RHO GTPase \\
\hline DLG-1 & VIT-5 & 0.60 & 0.00 & 1.00 & 0.33 & Vitellogenin \\
\hline DLG-1 & UNC-87 & 1.00 & 0.54 & 0.36 & 0.61 & Maintains structure of myofilaments \\
\hline DLG-1 & DLG-1 & 1.00 & 1.00 & 1.00 & 1.00 & Discs large \\
\hline DLG-1 & VIT-6 & 0.65 & 0.00 & 0.99 & 0.33 & Vitellogenin \\
\hline DLG-1 & ATAD-3 & 0.99 & 0.46 & 0.71 & 0.49 & Mitochondrial protein ATAD3 \\
\hline DLG-1 & DIM-1 & 0.22 & 0.00 & 0.04 & 0.98 & Uncharacterized \\
\hline DLG-1 & $\mathrm{C} 39 \mathrm{H} 7.4$ & 0.65 & - & 0.98 & 0.33 & Uncharacterized \\
\hline DLG-1 & COR-1 & 0.97 & 0.33 & 0.33 & 0.33 & Coronin \\
\hline DLG-1 & LEC-1 & 0.96 & 0.06 & 0.84 & 0.62 & Tandem-repeat type galectin \\
\hline DLG-1 & MAPH-1.1 & 0.00 & - & - & 0.93 & MAP1 homolog \\
\hline DLG-1 & IFB-2 & 0.66 & 0.00 & 0.90 & 0.93 & Intermediate filament protein \\
\hline DLG-1 & $\mathrm{F} 49 \mathrm{H} 12.5$ & 0.56 & 0.03 & 0.01 & 0.93 & Thioredoxin domain containing 12 \\
\hline DLG-1 & LARP-1 & 0.00 & 0.00 & 0.21 & 0.92 & La-related protein \\
\hline DLG-1 & IFC-2 & 0.00 & 0.00 & 0.91 & 0.33 & Intermediate filament protein \\
\hline DLG-1 & LEC-2 & 0.90 & 0.00 & 0.91 & - & Tandem-repeat type galectin \\
\hline DLG-1 & $\mathrm{R} 09 \mathrm{H} 10.5$ & 0.89 & 0.25 & 0.91 & 0.32 & Uncharacterized \\
\hline DLG-1 & MUP-2 & 0.90 & 0.63 & 0.28 & 0.66 & Muscle contractile protein troponin $T$ \\
\hline DLG-1 & ATN-1 & - & 0.31 & 0.90 & - & Alpha-actinin homolog \\
\hline DLG-1 & GPD-2 & 0.83 & 0.01 & 0.89 & 0.39 & GAPDH \\
\hline DLG-1 & QARS-1 & 0.00 & 0.00 & 0.88 & 0.39 & Glutaminyl (Q) tRNA synthetase \\
\hline DLG-1 & VARS-2 & 0.00 & - & 0.00 & 0.88 & Valyl-tRNA synthetase \\
\hline DLG-1 & NMT-1 & 0.00 & - & 0.58 & 0.87 & $\mathrm{~N}$-myristoyl transferase \\
\hline DLG-1 & MRG-1 & 0.00 & 0.00 & 0.12 & 0.87 & Homolog of MRG15 \\
\hline DLG-1 & EPS-8 & 0.85 & 0.05 & 0.82 & 0.66 & Cell signaling adaptor protein \\
\hline DLG-1 & T25F10.6 & 0.83 & 0.64 & 0.22 & 0.33 & Calponin \\
\hline DLG-1 & LEC-4 & 0.65 & - & 0.81 & 0.33 & Tandem-repeat type galectin \\
\hline LET-413 & LET-413 & 1.00 & 0.86 & 1.00 & - & Scribble \\
\hline LET-413 & B0303.3 & 0.82 & 0.00 & - & - & Beta-ketothiolase \\
\hline LET-413 & QARS-1 & 0.82 & 0.01 & - & - & Glutaminyl (Q) tRNA synthetase \\
\hline LGL-1 & LGL-1 & 1.00 & 1.00 & 1.00 & - & Lethal giant larvae (Lgl) \\
\hline LGL-1 & PAR-6 & 0.99 & 1.00 & 0.98 & - & Par-6 \\
\hline LGL-1 & PKC-3 & 0.98 & 0.97 & 0.91 & - & Atypical protein kinase $C$ \\
\hline LGL-1 & QARS-1 & 0.82 & 0.00 & 0.00 & - & Glutaminyl (Q) tRNA synthetase \\
\hline
\end{tabular}

Shown are putative interactors identified with a SAINT confidence score $>0.8$ in at least one of the four tissues examined. Ribosomal proteins are not shown. An expanded copy of this table including spectra counts is shown in Additional file 5: Table S2

embryos (Fig. 6c). In contrast, truncation of the C-terminal ETAV motif of ATAD-3 completely prevented the DLG-1 interaction in vivo (Fig. 6c). These data strongly support the presence of a direct physical interaction between ATAD-3 and DLG-1, mediated by the second PDZ domain of DLG-1 and the C-terminal ETAV motif of ATAD-3.

Both the ATAD- $3^{\mathrm{FL}}$ and the $\mathrm{ATAD}^{\mathrm{ETAV}}$ strains are viable. However, $\mathrm{ATAD}^{\triangle \mathrm{ETAV}}$ animals displayed a dramatically reduced fertility compared to wild type, as well as an increase in embryonic lethality (Fig. 6d). This phenotype was exacerbated at $25^{\circ} \mathrm{C}$ compared to $15^{\circ} \mathrm{C}$. These findings indicate that the interaction between ATAD- 3 and DLG-1 is physiologically relevant.

\section{Identification of a C. elegans MAP1-family member as a candidate neuron-specific DLG-1 interacting protein}

Four of the DLG-1 interactors co-purified exclusively with DLG-1::GTA biotinylated in neurons, and may therefore 
a

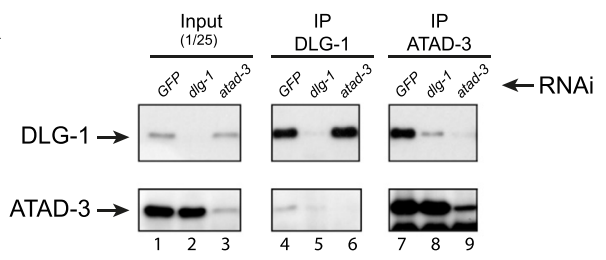

C

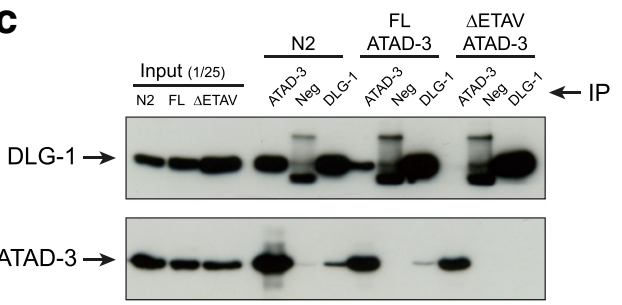

d

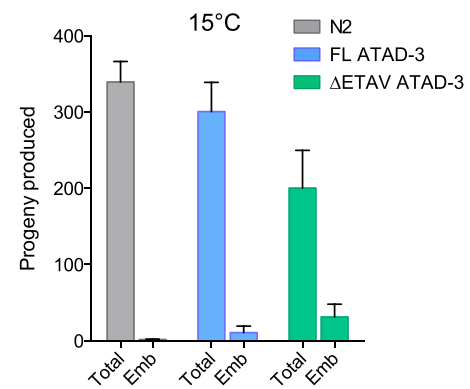

b

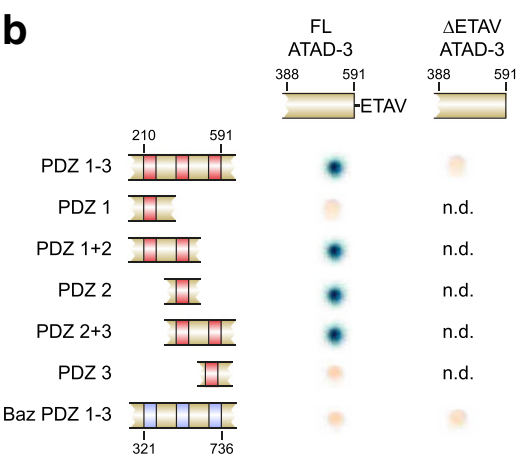

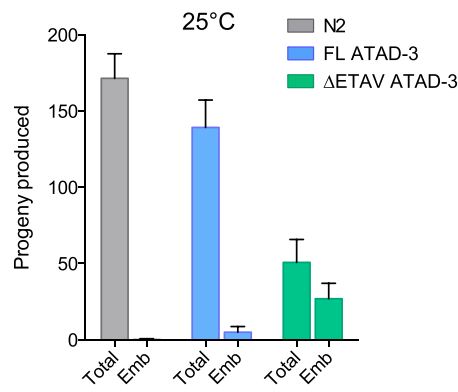

Fig. 6 Confirmation of the DLG-1/ATAD-3 interaction and mapping of the interaction domain. a Western blots showing co-immunoprecipitation (IP) of DGL-1 and ATAD-3. To demonstrate specificity of the detection, samples were treated with control RNAi (GFP RNAi) or RNAi against dlg-1 or atad-3. Left panels: input lysates before IP. Middle panels: DLG-1 IP. Right panels: ATAD-3 IP. b ATAD-3 interacts with DLG-1 in yeast two-hybrid experiments. This interaction depends on the C-terminal ETAV motif of ATAD-3 and the second PDZ domain of DLG-1. Note that ATAD-3 fragments lacking this C-terminal ETAV motif fail to interact with DLG-1 PDZ domains. A fragment containing PDZ 1-3 domains from Drosophila Bazooka was used as a negative control. n.d. not determined. c IP/western blotting experiment shows that, in vivo, the DLG-1/ ATAD-3 interaction requires the C-terminal ETAV motif of ATAD-3. Lanes on the left show input signals of DLG-1 and ATAD-3. The lanes on the right show IPs in corresponding lysates. d Progeny produced by N2 animals, or animals expressing full-length ATAD-3 (ATAD-3 ${ }^{F L}$ ) and ATAD-3 lacking the C-terminal four amino acids (ATAD ${ }^{\triangle E T A V}$ ) at $15^{\circ} \mathrm{C}$ and $25^{\circ} \mathrm{C}$. Total: average total progeny produced. Emb: average number of embryonic lethal progeny produced. Bars represent average values, and error bars the standard deviation. $n=4$

represent proteins that function with DLG-1 specifically in this tissue. BLAST searches with one of these proteins, F32A7.5, identified the MAP1 family of microtubuleassociated proteins (MAPs) as the closest homologs of F32A7.5. Mammalian genomes generally encode three MAP1 proteins: MAP1A, MAP1B, and the shorter family member MAP1S. In Drosophila, a single homolog termed Futsch has been described, which shares homology with mammalian MAP1A and MAP1B in the $\mathrm{N}$ - and C-terminal regions [47]. Interestingly, MAP1A localizes to postsynaptic densities, and interacts with the DLG-1 homologs DLG1 (SAP-97), DLG2 (PSD-93), and DLG4 (PSD-95) [48, 49]. To date, no C. elegans MAP1-family proteins have been described. We hypothesized that F32A7.5 represents a C. elegans MAP1-family member, and examined this protein in more detail.

MAP1A, MAP1B, and Futsch are large proteins (predicted molecular weights $305 \mathrm{kDa}, 270 \mathrm{kDa}$, and $592 \mathrm{kDa}$, respectively) with no predicted folded domains and extensive predicted unstructured regions. All three proteins are proteolytically cleaved into a heavy chain and a 26-30 kDa light chain derived from the C-terminus [50]. C. elegans F32A7.5 is smaller (predicted molecular weight before cleavage $92 \mathrm{kDa}$ ), but also contains a large predicted unstructured region. Analogous to Futsch, the similarity of F32A7.5 with the mammalian MAP1 proteins was largely limited to the $\mathrm{N}$ - and $\mathrm{C}$-terminus (Fig. 7a). Phylogenetic analysis indicated that F32A7.5 is equally related to MAP1A, MAP1B, and MAP1S (Fig. 7b and Additional file 8: Figure S5). The C. elegans genome encodes two other proteins that are highly similar to F32A7.5, termed F25D7.4 and C36A4.5. The high degree of similarity between these proteins ( $>85 \%$ amino acid identity) indicates that they are paralogs, and in the EggNOG 4.1 database of orthologous groups all three proteins are placed in the same orthologous group as mammalian MAP1A, MAP1B, and MAP1S [51]. Based on phylogeny and the results presented below, we name these proteins 


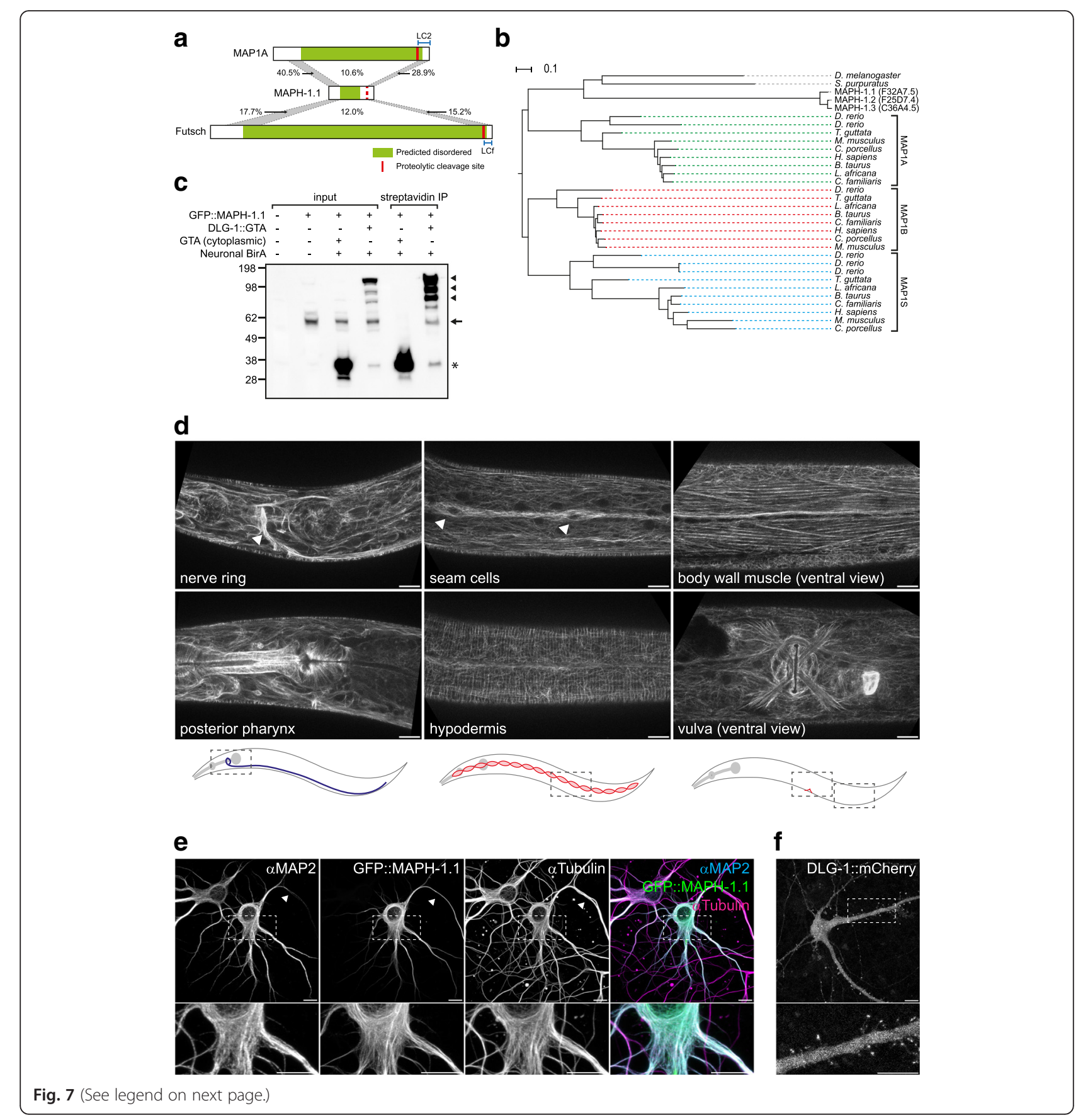




\begin{abstract}
(See figure on previous page.)
Fig. $7 \mathrm{MAPH}-1.1$ is a microtubule-associated protein related to mammalian MAP1 proteins. a Sequence similarity between MAPH-1.1 and human MAP1A, and between MAPH-1.1 and Drosophila Futsch. For each protein the predicted disordered region is indicated in green. Protein sizes are to scale. For MAP1A and Futsch, a red line indicates the proteolytic cleavage site that is used to generate a light chain (LC2 and LCf). A dotted red line indicates the homologous position in MAPH-1.1. Sequence similarity is indicated for three regions: two conserved $\mathrm{N}$ - and C-terminal regions (indicated in gray) and the intervening less conserved region. Amino acid coordinates for these regions are: MAP-1 N-terminal region 274-544, MAP-1 C-terminal region 2847-3041, MAPH-1.1 N-terminal region 1-230, MAPH-1.1 Cterminal region 742-878, Futsch N-terminal region 291-956, and Futsch C-terminal region 5082-5495. b Phylogenetic tree of MAP1-related proteins. Color coding indicates groups containing human MAP1A (green), MAP1B (red), and MAP1S (blue). c Western blots showing co-immunoprecipitation of DGL-1::GTA and GFP::MAPH-1.1. Input lysates (150 $\mu \mathrm{g}$ protein, lanes 1-4) show expression of DLG-1::GTA (arrowheads), GFP::MAPH-1 (arrow), and the empty GTA tag (asterisk). MAPH-1.1 co-purifies with DLG-1::GTA (lane 6) but not with the GTA tag alone (lane 5). Strains used are N2 (lane 1), BOX188 (lane 2), BOX209 (lanes 3 and 5), BOX212 (lanes 4 and 6). d Expression of GFP::MAPH-1 from an endogenously tagged GFP:::maph-1.1 locus. Nerve ring, pharynx, seam cells, and hypodermis panels were taken from the same $L 4$ stage animal. Body wall muscle and vulva panels are ventral views from a second animal. Arrowheads indicate the nerve ring an two seam cells. Cartoons are schematic representations of the areas imaged. e Expression of an N-terminal GFP::MAPH-1.1 fusion in 6-day-old primary rat hippocampal neuron cultures. Cells were co-stained for MAP2, which localizes to microtubules in dendrites, and for a-tubulin. The right panel is a merge with MAP2 in cyan, MAPH-1.1 in green, and microtubules in magenta. The arrowhead indicates the axon. f Expression of a C-terminal DLG-1:.mCherry fusion in 24-day-old primary hippocampal neuron cultures. All scale bars are $10 \mu \mathrm{m}$
\end{abstract}

MAPH-1.1, MAPH-1.2, and MAPH-1.3, for "microtubule-associated protein 1 homolog $1 . "$

To examine the expression pattern and subcellular localization of MAPH-1.1, and to validate the interaction with DLG-1, we used CRISPR/Cas9-based genome editing to engineer a GFP-tagged maph-1 locus (GFP::maph-1.1). By western blot, GFP::MAPH-1.1 has an apparent molecular weight of $\sim 60 \mathrm{kDa}$, much lower than the predicted $119 \mathrm{kDa}$ molecular weight of an unprocessed GFP::MAPH-1.1 fusion (Fig. 7c lane 2). This indicates that, like the mammalian Map1 proteins, MAPH-1.1 is proteolytically cleaved. To confirm the coaffinity purification of MAPH-1.1 with DLG-1, we crossed the GFP::maph-1.1 strain with a strain expressing DLG-1::GTA from its own promoter and BirA from the neuronal rgef-1 promoter. As a control, we crossed the GFP::maph-1.1 strain with a strain ubiquitously expressing cytoplasmic GFP::TEV::Avi, and expressing BirA in neurons. We purified DLG-1::GTA using streptavidin-coated beads, and tested for co-purification of MAPH-1.1 by western blotting using an antibody detecting GFP. We observed co-purification of MAPH-1.1 with DLG-1::GTA, but not with the GFP::TEV::Avi control (Fig. 7c). This confirms the identification of MAPH1.1 in neuronal DLG-1 purifications by MS.

We observed broad expression of GFP::MAPH-1.1 in multiple somatic postembryonic tissues. This included neurons, but also body wall muscle cells, the vulva, vulval muscle cells, the hypodermis, seam cells, and intestinal cells (Fig. 7d). The fact that we did not identify MAPH-1.1 in DLG-1 purifications from seam or intestinal cells implies that this interaction is either occurring only in neurons, or stabilized in neurons by a neuronspecific protein.

The subcellular localization pattern of GFP::MAPH-1.1 closely resembled that of previously described noncentrosomal microtubule arrays in uterine muscle cells [52], circumferential microtubule arrays in the hypodermis
[53], and body wall muscle cells [54] (Fig. 7d). Furthermore, live imaging revealed a highly dynamic behavior of the observed arrays, including shrinkage events (Additional file 9: Movie S1; Additional file 10: Movie S2; Additional file 11: Movie S3; Additional file 12: Movie S4; Additional file 13: Movie S5; and Additional file 14: Movie S6). We measured the shrinkage rate of several arrays in the hypodermis and body wall muscle cells, and obtained an average rate of $0.76 \mu \mathrm{m} / \mathrm{s}$ and $0.85 \pm 0.14 \mu \mathrm{m} / \mathrm{s}$ respectively (Additional file 15: Figure S6). These rates are consistent with the previously observed shrinkage rate of $0.85 \mu \mathrm{m} / \mathrm{s}$ for non-centrosomal microtubule bundles in differentiated uterine muscle cells [52]. Finally, we examined the dynamics of GFP::MAPH-1.1 using fluorescence recovery after photobleaching (FRAP). GFP::MAPH-1.1 showed a half-time of recovery of $6 \mathrm{~s}$, which is similar to the rapid dynamics observed for the microtubule-binding domain of ensconsin (MAP7) [55]. All of these observations are consistent with MAPH-1.1 acting as a microtubulebinding protein. Nevertheless, in body wall muscle cells and the hypodermids, tubulin and actin can form bundles that are similar in appearance. Given that mammalian MAP1 proteins can associate with actin [50], we cannot exclude that MAPH-1.1 also localizes to actin structures.

To further confirm the localization of MAPH-1.1 to microtubules, particularly in neurons, we expressed a translational GFP::MAPH-1.1 fusion protein in cultured primary rat hippocampal neurons, in which microtubules are easily visualized. Co-staining with an antibody directed against $\alpha$-tubulin showed clear co-localization of MAPH-1.1 with microtubules in the neuronal cell body, axon, and dendrites (Fig. 7e). We also expressed a DLG-1:: mCherry fusion protein in cultured hippocampal neurons, and observed localization to synaptic structures in dendrites (Fig. 7f). We conclude that, like mammalian MAP1A, MAPH-1.1 is a microtubuleassociated protein, and a candidate neuron-specific interaction partner of DLG-1. In contrast to MAP1A, 
however, maph-1.1 is broadly, if not ubiquitously, expressed.

\section{Discussion}

Here, we describe an in vivo biotinylation-based AP/MS approach to purify protein complexes from specific tissues in C. elegans. Proteins of interest were expressed with a GTA tag under the control of their normal regulatory sequences, and biotinylation in a single tissue was accomplished by expressing BirA from a tissue-specific promoter. Expression of GFP::TEV::Avi and BirA in two different tissues confirmed that biotinylation and purification are indeed tissue-specific. The ability of our approach to identify bona fide protein interactions from specific tissues was demonstrated by the identification of the known LGL-1 interaction partners PAR- 6 and PKC3 from two distinct epithelial tissues. We also detected a prominent interaction between the junctional scaffold protein DLG-1 and the mitochondrial AAA ATPase ATAD-3. We confirmed this interaction through affinity purification, identified the interacting motif by yeast two-hybrid assays, and demonstrate the requirement for this motif for the ATAD-3/DLG-1 interaction in vivo. Finally, through neuron-specific purification of DLG-1 we identified MAPH-1.1 as an interactor of DLG-1, and demonstrate that this protein represents a $C$. elegans member of the MAP1 family of MAPs.

As is generally the case with interaction detection assays, not all known interactions were recovered. In some cases, this may have been due to the stringency of the SAINT threshold of 0.8 , as potentially relevant interactions were observed below this threshold. For example, AJM-1 is a known interaction partner of DLG-1 [31], and co-purified with DLG-1 from neurons with a SAINT score of 0.33. Similarly, in MDCK cells, Scribble has been reported to associate with Lgl2 [56], and in our experiments LGL-1 co-purified with LET-413 from intestine with a SAINT score of 0.33. Neither AJM-1 nor LGL-1 co-purified with other baits, which indicates that these may represent valid and specific interactions. We also observed that not all bait proteins were recovered at equal levels, which may contribute to the lack of recovery of some known interaction partners. A number of different reasons may cause inefficient bait protein recovery. For example, some proteins may be present at low levels, or may not be effectively released in soluble form into the supernatant by the lysis procedure. It is also possible that some proteins are biotinylated less efficiently in vivo, or that the efficiency of TEV cleavage varies. In part, such issues may be overcome by optimization of lysis and purification conditions for specific bait proteins.

An affinity purification tag incorporating GFP and Avi has been used previously in C. elegans to purify a protein complex [19]. However, tissue specificity of the biotinylation and purification procedure had not been demonstrated, and no tissue-specific protein interactions had been identified to date. Two other proteomics approaches using BirA-mediated in vivo biotinylation have also been described in C. elegans. One study used in vivo biotinylation of the histone $\mathrm{H} 3.3$ protein for the purification of chromatin and epigenetic profiling [57]. Another study used biotinylation of the nuclear pore complex component NPP-9 to purify nuclei from specific tissues [58]. Thus, in vivo biotinylation is proving to be a versatile method for tissue-specific proteomics approaches in C. elegans.

The GTA tag includes GFP to visualize the subcellular localization of tagged proteins. The expression patterns of the GTA-tagged proteins we observed largely confirmed known localizations, though we failed to observe intestinal localization of PAR-3::GTA. Possibly, the regulatory sequences of PAR-3 extend beyond the region included in the fosmid, or the par-3 splice variants we tagged are not expressed in the intestine. Two alternative start sites have already been documented to affect the expression pattern of par-3, and it is conceivable that different 3 '-ends can also affect par-3 expression [59]. In addition to previously described localization patterns, we observed expression of DLG-1::GTA in ventral cord neurons. Although the localization did not clearly resemble a synaptic pattern, this observation is consistent with the identification of mammalian DLG-1 homologs as postsynaptic density (PSD) proteins.

We identified a novel interaction between the junctional scaffold protein DLG-1 and the evolutionarily conserved mitochondrial AAA ATPase ATAD-3. ATAD-3 and its homologs in other species (ATAD3A, ATAD3B) have been described as mitochondrial proteins with roles in tumor progression [44, 60-63]. Inactivation of atad-3 by RNAi causes defects in the development and functioning of mitochondria, and results in embryonic lethality and early larval arrest [44]. Immunopurification followed by western blotting indicated that a substantial fraction of DLG-1 associates with ATAD-3, while only a small sub-fraction of ATAD-3 is in complex with DLG-1. ATAD-3 contains a putative transmembrane domain and has been reported to be inserted into the inner membrane of mitochondria, with its $\mathrm{C}$-terminus located in the matrix and the $\mathrm{N}$-terminus facing the cytosol $[61,64,65]$. Given the specific binding of DLG-1 to the C-terminal end of ATAD-3, the DLG-1/ ATAD-3 interaction should either occur in the matrix of mitochondria, or involve a subpopulation of ATAD-3 that is not present in the mitochondria. Results from several studies support that mammalian ATAD3A does not reside exclusively in mitochondria $[60,66,67]$. In fact, a substantial number of mitochondrial proteins are dually localized [68]. In particular, proteins that have a less well-defined 
mitochondrial targeting sequence are more likely to be found at multiple subcellular locations. ATAD-3 has no recognizable mitochondrial targeting sequence and has been found in the plasma-membrane in certain cancer cells [67], and in the endoplasmic reticulum mitochondriaassociated membrane (MAM) fraction [60]. These findings indicate the possibility for interactions with ATAD-3 outside of mitochondria. Our finding that expression of ATAD- ${ }^{\mathrm{FL}}$ rescues the atad-3(ok3093) mutation to a greater extent than expression of ATAD ${ }^{\triangle \mathrm{ETAV}}$ suggests that the DLG-1/ATAD-3 interaction plays an important role in C. elegans development. In epithelial cells, DLG-1 localizes to cell junctions [69], a location that has not been observed for ATAD-3. Although the exact contribution of the interaction with ATAD-3 to DLG-1 remains to be discovered, it is possible that this interaction is of importance before the formation of cell junctions, or affects a non-junctional pool of DLG-1.

MAPs control the organization, stability, and function of the microtubule cytoskeleton [50]. The mammalian MAP1 family of MAPs contains three family members: MAP1A, MAP1B, and the smaller MAP1S [50]. In Drosophila, a single MAP1 protein, termed Futsch, has been identified [47]. MAP1A, MAP1B, and Drosophila Futsch are predominantly expressed in neurons, and have been shown to play a role in several aspects of neuronal development and functioning [47, 50]. MAP-1A is found in PSDs, where it interacts with the mammalian DLG1 (SAP-97), DLG2 (PSD-93), and DLG4 (PSD-95) [48, 49]. To date, no $C$. elegans MAP1 proteins had been described, and no clear orthologs are present in the genome. Purification of C. elegans DLG-1 from neurons resulted in the identification of F32A7.5 as a candidate interaction partner. Based on sequence similarity to mammalian MAP1 proteins, we postulate that F32A7.5 is a $C$. elegans MAP1 protein, which we named MAPH1.1 for "microtubule-associated protein 1 homolog 1." MAPH-1.1 did not co-purify with DLG-1 biotinylated in the intestine or epidermal cells, nor with ubiquitously biotinylated DLG-1, despite expression of MAPH-1.1 in many non-neuronal tissues, including seam and intestine. Despite the high SAINT score with which MAPH1.1 was identified in neuron-specific DLG-1 purifications, the actual number of peptides identified was low. This may reflect that only a minor fraction of DLG-1 and MAPH-1.1 associate in neurons, or that a neuron-specific protein stabilizes the DLG-1/MAPH-1.1 interaction, which would illustrate the value of using a tissue-specific approach. However, further study will be needed to confirm a biological role of this interaction in neurons in vivo.

The localization of MAPH-1.1 to dynamic arrays resembling previously published microtubule arrays, the shrinkage parameters of these arrays, and the colocalization with microtubules in cultured rat primary hippocampal neurons all support a function for MAPH1.1 as a MAP. The $C$. elegans genome encodes two other proteins that are highly similar to F32A7.5, termed F25D7.4, and C36A4.5, which we termed MAPH-1.2 and MAPH-1.3. We did not identify MAPH-1.2 in any of our purifications, but MAPH-1.3 also interacted with DLG-1 specifically in neurons, though at a much lower confidence level (SAINT score 0.33).

MAP1A binds to the guanylate kinase (GK) domain of DLG4 through a short peptide motif [49], which does not appear to be conserved in the C. elegans MAPH-1 proteins. However, mutational analysis of the GK binding motif showed that it is highly permissive of changes, and GK binding sites do not appear to be well conserved across different proteins [49]. Thus, it is possible that the mammalian consensus sequence has diverged from the MAPH-1.1 binding site for DLG-1. Mammalian MAP1A and MAP1B, as well as Futsch, are proteolytically cleaved into heavy and light chains [50, 70]. Residues surrounding the cleavage site are poorly conserved between MAP1A/B and Futsch, and we could not identify a candidate cleavage site in MAPH-1.1 by sequence analysis. Thus, it remains to be determined if the C. elegans MAPH-1 proteins are proteolytically cleaved. In mammals, MAP1A and MAP1B heavy and light chains assemble into a trimeric complex together with the separately encoded MAP1 light chain 3 (LC3) [50]. The C. elegans protein LGG-2, which plays an important role in the generation of autolysosomes, is highly similar to LC3 [71], consistent with conservation of the mechanism of MAP1 functioning in C. elegans. However, a role for LGG-2 in neuronal function or microtubule regulation has not been examined.

\section{Conclusions}

The approach we presented here should be widely applicable for the identification of protein complexes for many proteins, provided that the bait protein is soluble after lysis and can be tagged at either the $\mathrm{N}$ terminus or C-terminus without interfering with the function of the protein. In addition, our approach can be used to identify tissue-specific protein modifications of the bait protein, such as phosphorylations, and to reveal differences in protein complex composition over time by using synchronized cultures. Here, we used fosmid-based recombineering to generate the transgenic strains. With the development of CRISPR/ Cas9-based genome engineering, it has become possible to endogenously tag any $C$. elegans gene of interest [72]. Thus, genes can now be endogenously tagged with the GTA tag, which eliminates the need for crosses with a null mutant background, and should result in even more reliable expression patterns. 


\section{Methods}

\section{Cloning of GTA tags}

To generate the $\mathrm{N}$ - and C-terminal GTA tag constructs pMB41 and pMB72, we inserted TEV and Avi sequences obtained as synthetic DNA constructs from GenScript (http://www.genscript.com), and codon-optimized GFP/ GalK sequences from vector pBALU1 [26] into vector pUC19 (NEB, http://www.neb.com) by Gibson assembly. Vector maps and sequences are available in Additional file 16. pMB41 and pMB72 are available through Addgene (see "Availability of data and materials" below).

\section{Recombineering}

The recombineering approach we used has been described previously [26]. Briefly, the fosmid to be engineered is transformed into E. coli strain SW105, a galKdefective strain carrying a heat shock-inducible phage $\lambda$ Red recombinase, and an arabinose-inducible Flp recombinase. Bacteria carrying the fosmid are transformed with a PCR product consisting of the tag to be introduced, which also carries the wild-type galK sequence, flanked on both sides by 50 nucleotides identical to the insertion site. Expression of $\lambda$ Red induces homologous recombination between the fosmid and the PCR product, and successful recombinants are selected on media with galactose as the only carbon source. Finally, galK sequences, which are flanked by FRT sites, are eliminated from the fosmid by expression of the Flp recombinase.

We used the following fosmids and primers to generate the PCR products used as templates for homologous recombination: For tagging $d l g-1$ we used the C-terminal tag, fosmid WRM067dB05, and primers rec_dlg-1_C-term_F (5' -actccatcatcagccgtgaatcgcagacgccaatttgggtgccacgtcatgga ggaggatctggaggaggaggatctggaggagga) and rec_dlg-1_C-te rm_R (5' -acatatttcttgaagaaacgattatttgtctaaaaaatatccaatttcat ctattcatgccattcaatcttctgagcttcg); for let-413 the C-terminal tag, fosmid WRM0640dF02, and primers rec_let-413_Cterm_F (5' -ggtcccatcgccagtttcgagaacatctgtgagtaggccatgtg agtatggaggaggatctggaggaggaggatctggaggagga) and rec_let413_C-term_R (5' -gaatgtcaaaaaaaaaacgtctaatgtctagttttcagc caaaatcggcctcattcatgccattcaatcttctgagcttcg); for $l g l-1$ the Cterminal tag, fosmid WRM065bB11, and primers rec_lgl1_C-term_F (5' -gaagtacggtgaatttgaactttcgcggttggagcagtacg cacaagtcaggaggaggatctggaggaggaggatctggaggagga) and rec _lgl-1_C-term_R (5' -aaaattaatatatatcaacaggaaaacgatttttaa aaaaaatgcatctattcatgccattcaatcttctgagcttcg); for par-3 the Cterminal tag, fosmid WRM064bG02, and primers rec_par3_C-term_F (5' -gccaataccgtcgcagagatcagggaccgcctcatcgtt ttcccagtacggaggaggatctggaggaggaggatctggaggagga) and rec_par-3_C-term_R (5'-gattccgtatttttcgcggctgcgtaatataac tttgagaaaaaactgacctattcatgccattcaatcttctgagcttcg); and for cdc-42 the N-terminal tag, fosmid WRM0612bG08, and primers rec_cdc-42_N-term_F (5' -ctataaagacgtaattttaat acttttattcatttttttttcaggcgaaaaaatgggacttaatgatattttcgaagctc ag) and rec_cdc-42_N-term_R (5' -gttttaccgacagctccatctc caacgacgacgcacttgatcgtctgcatacctcctcctccagatcctcctcct). To check whether the recombineering procedure was successful we performed a PCR reaction on the constructs with primers rec_dlg-1_C-term_check_F (5'-aagctcaagcgcagt attcc) and rec_dlg-1_C-term_check_R (5' -tttcttgaattgagaa cttggaaa) for $d l g-1$, primers rec_let-413_C-term_check_F (5' -cgattggtattccgattggt) and rec_let-413_C-term_check_R (5'-gccgaacagtaacggagatt) for let-413, primers rec_lgl-1_Cterm_check_F (5' -gggagttatgtacaggcatctagta) and rec_lgl1_C-term_check_R (5'-taagccagccgctagcac) for lgl-1, primers rec_par-3_C-term_check_F (5'-tatgccgcgaaggagaa gta) and rec_par-3_C-term_check_R (5'-ttcgctcagcggaatt atc) for par-3, and primers rec_cdc-42_N-term_check_F (5'-tcgtttattaaggcgtttaccg) and rec_cdc-42_N-term_check _R ( $5^{\prime}$-cgatcgtaatcttcctgtcc) for $c d c-42$.

\section{Cloning the BirA constructs}

The C. elegans codon-optimized BirA sequence was obtained as a synthetic DNA construct from GenScript (http://www.genscript.com). To generate the BirA expression constructs pMB37 (Prps-27::BirA), pMB71 (Pelt2::BirA), and pMB73 (Pwrt-2::BirA), the BirA sequence was cloned into vector pPD158.87 (Addgene \#1709) using KpnI and EcoRI restriction sites. For vector pBT331 (Prgef-1::BirA), the BirA sequence was cloned into vector backbone pPD95.77 (Addgene plasmid \#1495) using KpnI and EcoRI. Next, promoter regions were amplified from C. elegans genomic DNA and cloned into appropriate restriction sites. For pMB37, the rps-27 promoter was amplified using primers Prps-27F (5'-aaa CTGCAGttcaatcggtttttccttgcttgc) and Prps-27R (5' -aaaGGTACCattcc acttgttgagcggggctg), and cloned using PstI/KpnI. For pMB71, the elt-2 promoter was amplified using primers Pelt-2F (5'-aaaCTGCAGtaatttcgaaatgtatgaactccaattc) and Pelt-2R (5' -aaaCCCGGGctataatctattttctagtttc), and cloned using PstI/SmaI. For pMB73, the wrt-2 promoter was amplified using primers Pwrt-2F (5'-aaaCTGCAGcaggtcg actccacgtaatttc) and Pwrt-2R (5' -aaaCCCGGGGATCCccg agaaacaattggcaggttg), and cloned using PstI/SmaI. For pBT331, the rgef-1 promoter was amplified using primers Prgef-1F (5'- aaCTGCAGcgtttccgataccccttatatc) and Prgef-1R (5'-aaaCCCGGGgatcctttactgctgatcgtcg), and cloned using PstI/SmaI. Vector maps and sequences are available in Additional file 16. The BirA expression constructs are available through Addgene (see "Availability of data and materials" below).

\section{Cloning the control constructs}

To generate expression constructs pMB43 (Prps27::GTA), pMB76 (Pelt-2::GTA), and pMB77 (Pwrt$2:: G T A)$, we first removed the GalK sequences from the GTA tag vector pMB71 by expression of Flp recombinase. The resulting GFP-2xTEV-Avi sequence was then 
amplified using primers GTA_F (5'-aaaGGTACCggtagaaaaaatgagtaaaggagaagaacttttc) and GTA_R (5'-aaaGCTAGCttattcatgccattcaatcttctgag), and cloned into vector pPD158.87 (Addgene \#1709) using KpnI and NheI. Next, promoter regions were amplified from $C$. elegans genomic DNA and cloned into appropriate restriction sites. For pMB43, the rps-27 promoter was amplified using primers Prps-27F (5'-aaaCTGCAGttcaatcggtttttcctt gcttgc) and Prps-27R (5'-aaaGGTACCattccacttgttgag cggggctg), and cloned using PstI/KpnI. For pMB76, the elt-2 promoter was amplified using primers Pelt-2F (5'aaaCTGCAGtaatttcgaaatgtatgaactccaattc) and Pelt-2R (5'-aaaCCCGGGctataatctattttctagtttc), and cloned using PstI/SmaI. For pMB77, the wrt-2 promoter was amplified using primers Pwrt-2F (5'-aaaCTGCAGcaggtcgactccacgtaatttc) and Pwrt-2R (5'-aaaCCCGGGGATCCccgagaaac attggcaggttg), and cloned using PstI/SmaI. For pMB43, the let-858 3'-UTR was replaced with the tbb-2 3'UTR by PCR amplifying the $t b b-23^{\prime}$-UTR with primers tbb-2U_F (5'-aaaGCTAGCatgcaagatcctttcaagc) and tbb2U_R (5'-aaaGGGCCCtgatccacgatctggaagatttc), and cloning using NheI and ApaI restriction sites. Vector maps and sequences are available in Additional file 16.

\section{C. elegans strains and culture conditions}

Unless otherwise indicated, strains were maintained at $15{ }^{\circ} \mathrm{C}$ as previously described [73]. Transgenic strains were generated by injecting constructs into the gonad of young adult $\mathrm{N} 2$ animals using conventional micro-injection procedures. N2 animals were obtained from the Caenorhabditis Genetics Center. The amounts of each construct injected are indicated for each strain, and were supplemented to a final DNA concentration of $80 \mathrm{ng} / \mu \mathrm{l}$ with PstI digested phage $\lambda$ DNA. The resulting transgenic strains carrying extrachromosomal arrays were subjected to gamma irradiation to integrate the construct into the $C$. elegans genome. The following strains were generated:

BAT5: barIs3 [Prgef-1::BirA, Pceh-36::mCherry] X

BOX20: $\quad$ mibIs7[Pwrt-2::BirA $10 \quad n g / \mu l+$ Pmyo-

2::mCherry $2.5 \mathrm{ng} / \mu l] I I$

BOX27: mibIs14[Pelt-2::BirA $10 \quad n g / \mu l+$ Pmyo-

2::mCherry $2.5 \mathrm{ng} / \mu \mathrm{ll} I$

BOX41: mibIs23[lgl-1::GFP-2xTEV-Avi 10 ng/Ml, Pmyo3:: $m$ Cherry $5 \mathrm{ng} / \mathrm{\mu l}] \mathrm{V}$

BOX43: mibIs25[Avi-2xTEV-GFP::cdc-42 $10 \mathrm{ng} / \mu \mathrm{l}$,

Pmyo-3:: $m$ Cherry $5 \mathrm{ng} / \mu l] X$

BOX51: mibIs26[par-3::GFP-2xTEV-Avi $10 \mathrm{ng} / \mu l$,

Pmyo-3:: $m$ Cherry $5 \mathrm{ng} / \mathrm{\mu l}] \mathrm{V}$

BOX55: mibIs30[let-413::GFP-2xTEV-Avi $10 \mathrm{ng} / \mu l$,

Pmyo-3::mCherry $5 \mathrm{ng} / \mu l] X$

BOX56: mibIs31[dlg-1::GFP-2xTEV-Avi $10 \mathrm{ng} / \mu l$, Pmyo-3:: $m$ Cherry $5 \mathrm{ng} / \mu \mathrm{ll} \mathrm{V}$

BOX58: mibIs33[Prps-27::BirA $10 \mathrm{ng} / \mu l$, Pmyo2::mCherry $2.5 \mathrm{ng} / \mu \mathrm{ll} I$
BOX60: mibIs35 [Prgef-1::BirA $10 \quad n g / \mu l$, Pmyo-

2::mCherry $2.5 \mathrm{ng} / \mathrm{\mu l}] \mathrm{II}$

BOX61: mibIs36[Pwrt-2::GFP-2xTEV-Avi $10 \quad n g / \mu l$,

Prab-3::mCherry $5 \mathrm{ng} / \mu \mathrm{ll} X$

BOX62: mibIs37[Pelt-2::GFP-2xTEV-Avi $10 \mathrm{ng} / \mu l$,

Prab-3::mCherry $5 \mathrm{ng} / \mu \mathrm{ll} X$

BOX65: mibIs40[Prps-27::GFP-2xTEV-Avi $10 \mathrm{ng} / \mu \mathrm{l}$,

Prab-3::mCherry 5 ng/ $\mu l] I I I$

BOX99: mibIs14[Pelt-2::BirA $10 \quad n g / \mu l$, Pmyo-

2::mCherry 2.5 ng/ul]I; mibIs37[Pelt-2::GFP-2xTEV-Avi

$10 \mathrm{ng} / \mu l$, Prab-3::mCherry $5 \mathrm{ng} / \mu l] X$

BOX100: mibIs7[Pwrt-2::BirA $10 \mathrm{ng} / \mu \mathrm{l}$, Pmyo2::mCherry $2.5 \mathrm{ng} / \mu \mathrm{l}] \mathrm{II} ;$ mibIs37[Pelt-2::GFP-2xTEV-Avi $10 \mathrm{ng} / \mu \mathrm{l}$, Prab-3:: $m$ Cherry $5 \mathrm{ng} / \mu \mathrm{ll} X$

BOX101: mibIs14[Pelt-2::BirA $10 \mathrm{ng} / \mu \mathrm{l}$, Pmyo2::mCherry 2.5 ng/Ml]I; mibIs36[Pwrt-2::GFP-2xTEV-Avi $10 \mathrm{ng} / \mu \mathrm{l}$, Prab-3::mCherry $5 \mathrm{ng} / \mu \mathrm{ll} \mathrm{X}$

BOX102: mibIs7[Pwrt-2::BirA $10 \mathrm{ng} / \mu l$, Pmyo2::mCherry $2.5 \mathrm{ng} / \mu \mathrm{l}] I I ;$ mibIs36[Pwrt-2::GFP-2xTEV-Avi $10 \mathrm{ng} / \mu \mathrm{l}$, Prab-3::mCherry $5 \mathrm{ng} / \mu \mathrm{ll} \mathrm{X}$

BOX103: mibIs33[Prps-27::BirA $10 \mathrm{ng} / \mu \mathrm{l}$, Pmyo2::mCherry $2.5 \mathrm{ng} / \mu \mathrm{l}] \mathrm{I}$; mibIs26[par-3::GFP-2xTEV-Avi $10 \mathrm{ng} / \mu \mathrm{l}$, Pmyo-3:: mCherry $5 \mathrm{ng} / \mu \mathrm{ll} \mathrm{V}$

BOX104: mibIs7[Pwrt-2::BirA $10 \mathrm{ng} / \mu \mathrm{l}$, Pmyo2::mCherry $2.5 \mathrm{ng} / \mu l$ lII; mibIs26[par-3::GFP-2xTEV-Avi $10 \mathrm{ng} / \mu \mathrm{l}$, Pmyo-3:: mCherry $5 \mathrm{ng} / \mu \mathrm{ll} \mathrm{V}$

BOX105: mibIs14[Pelt-2::BirA $10 \mathrm{ng} / \mu \mathrm{l}$, Pmyo-

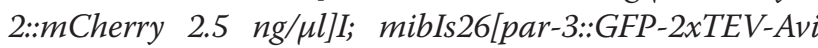
$10 \mathrm{ng} / \mu \mathrm{l}$, Pmyo-3::mCherry $5 \mathrm{ng} / \mu \mathrm{ll} \mathrm{V}$

BOX106: mibIs33[Prps-27::BirA $10 \mathrm{ng} / \mu \mathrm{l}$, Pmyo2::mCherry $2.5 \mathrm{ng} / \mu \mathrm{ll} I ; \quad$ mibIs31[dlg-1::GFP-2xTEV-Avi $10 \mathrm{ng} / \mu l$, Pmyo-3::mCherry $5 \mathrm{ng} / \mu l] V ;$ dlg-1(ok318)X

BOX107: mibIs7[Pwrt-2::BirA $10 \mathrm{ng} / \mu \mathrm{l}$, Pmyo2::mCherry $2.5 \mathrm{ng} / \mu l] I I ;$ mibIs31[dlg-1::GFP-2xTEV-Avi $10 \mathrm{ng} / \mu l$, Pmyo-3::mCherry $5 \mathrm{ng} / \mu l] \mathrm{V}$; dlg-1(ok318)X

BOX108: mibIs14[Pelt-2::BirA $10 \mathrm{ng} / \mu \mathrm{l}$, Pmyo2::mCherry 2.5 ng/ul]I; mibIs31[dlg-1::GFP-2xTEV-Avi

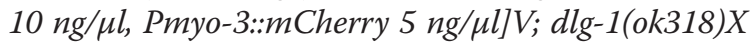

BOX109: mibIs35 [Prgef-1::BirA $10 \mathrm{ng} / \mu \mathrm{l}$, Pmyo2::mCherry $2.5 \mathrm{ng} / \mu \mathrm{l}$ ] II; mibIs31 [dlg-1::GFP-2xTEV-Avi $10 \mathrm{ng} / \mu \mathrm{l}$, Pmyo-3::mCherry $10 \mathrm{ng} / \mu \mathrm{ll} \mathrm{V}$; dlg-1(ok318) X

BOX110: mibIs33[Prps-27::BirA $10 \mathrm{ng} / \mu \mathrm{l}$, Pmyo-

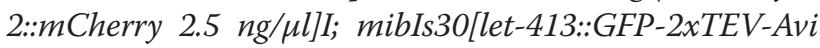
$10 \mathrm{ng} / \mu l$, Pmyo-3::mCherry $5 \mathrm{ng} / \mu l] X$

BOX111: mibIs7[Pwrt-2::BirA $10 \mathrm{ng} / \mu \mathrm{l}$, Pmyo2::mCherry $2.5 \mathrm{ng} / \mu \mathrm{l}] I I ;$ mibIs30[let-413::GFP-2xTEV-Avi 10 ng/ul, Pmyo-3::mCherry 5 ng/ul]X

BOX112: mibIs14[Pelt-2::BirA $10 \mathrm{ng} / \mu \mathrm{l}$, Pmyo2::mCherry $2.5 \mathrm{ng} / \mu \mathrm{ll}$ I; mibIs30[let-413::GFP-2xTEV-Avi $10 \mathrm{ng} / \mu l$, Pmyo-3::mCherry $5 \mathrm{ng} / \mu l] X$

BOX113: mibIs33[Prps-27::BirA $10 \mathrm{ng} / \mu \mathrm{l}$, Pmyo2::mCherry $2.5 \mathrm{ng} / \mu l] I ; \quad$ mibIs23[lgl-1::GFP-2xTEVAvi $10 \mathrm{ng} / \mu l$, Pmyo-3::mCherry $5 \mathrm{ng} / \mu \mathrm{ll} \mathrm{V}$; lgl1(tm2616)X 
BOX114: mibIs7[Pwrt-2::BirA $10 \quad n g / \mu l$, Pmyo2::mCherry $2.5 \mathrm{ng} / \mu l] I I ;$ mibIs23[lgl-1::GFP-2xTEV-Avi $10 \mathrm{ng} / \mu \mathrm{l}$, Pmyo-3:: mCherry $5 \mathrm{ng} / \mu \mathrm{ll} \mathrm{V}$; lgl-1(tm2616)X

BOX115: mibIs14[Pelt-2::BirA $10 \mathrm{ng} / \mu \mathrm{l}$, Pmyo2::mCherry $2.5 \mathrm{ng} / \mathrm{Ml}]$ I; mibIs23[lgl-1::GFP-2xTEV-Avi $10 \mathrm{ng} / \mu \mathrm{l}$, Pmyo-3::mCherry $5 \mathrm{ng} / \mu \mathrm{ll} \mathrm{V}$; $\operatorname{lgl}-1(\operatorname{tm} 2616) X$

BOX116: mibIs40[Prps-27::GFP-2xTEV-Avi $10 \mathrm{ng} / \mu l$,

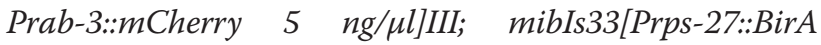
$10 \mathrm{ng} / \mu \mathrm{l}$, Pmyo-2::mCherry $2.5 \mathrm{ng} / \mu \mathrm{ll} \mathrm{I}$

BOX117: mibIs7[Pwrt-2::BirA $10 \mathrm{ng} / \mu \mathrm{l}$, Pmyo2:: mCherry $2.5 \mathrm{ng} / \mu \mathrm{ll} I I ;$ mibIs40[Prps-27::GFP-2xTEV-

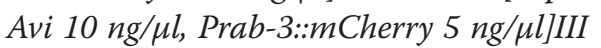

BOX118: mibIs14[Pelt-2::BirA $10 \mathrm{ng} / \mu \mathrm{l}$, Pmyo2::mCherry $2.5 \mathrm{ng} / \mu \mathrm{ll}$ I; mibIs40[Prps-27::GFP-2xTEV-Avi $10 \mathrm{ng} / \mu \mathrm{l}$, Prab-3::mCherry $5 \mathrm{ng} / \mu \mathrm{ll} I \mathrm{II}$

BOX119: mibIs35 [Prgef-1::BirA $10 \mathrm{ng} / \mu \mathrm{l}$, Pmyo2::mCherry $2.5 \mathrm{ng} / \mu \mathrm{l}] \mathrm{II}$; mibIs40 [Prps-27::GFP-2xTEVAvi 10 ng/ul, Prab-3::mCherry 5 ng/ul] III

BOX133: mibIs7 [Pwrt-2::BirA $10 \mathrm{ng} / \mu l$, Pmyo2::mCherry] II; mibIs25 [cdc-42::GFP-2TEV-Avi $10 \mathrm{ng} / \mu \mathrm{l}$, Pmyo-3::mCherry 10ngul] $X$

BOX134: mibIs33 [Prps-27::BirA $10 \mathrm{ng} / \mu \mathrm{l}$, Pmyo2:: $m$ Cherry $2.5 \mathrm{ng} / \mu \mathrm{l}$ ] I; mibIs25 [cdc-42::GFP-2TEV-Avi $10 \mathrm{ng} / \mu \mathrm{l}$, Pmyo-3::mCherry $10 \mathrm{ng} / \mu \mathrm{ll} \mathrm{X}$

BOX135: mibIs14 [Pelt-2::BirA $10 \mathrm{ng} / \mu l$, Pmyo2:: mCherry $10 \mathrm{ng} / \mu \mathrm{ll}$ I; mibIs25 [cdc-42::GFP-2TEV-Avi $10 \mathrm{ng} / \mu l$, Pmyo-3::mCherry $10 \mathrm{ng} / \mu \mathrm{ll} \mathrm{X}$

BOX188: maph-1.1(mib12[GFP::maph-1.1]) I

BOX209: maph-1.1(mib12[GFP::maph-1.1]) I; mibIs35 [Prgef-1::BirA $10 \mathrm{ng} / \mu \mathrm{l}$, Pmyo-2::mCherry $2.5 \mathrm{ng} / \mu \mathrm{l}] \mathrm{II}$;

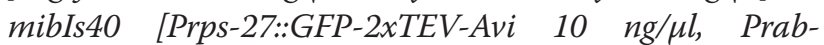
3::mCherry $5 \mathrm{ng} / \mathrm{\mu l}] \mathrm{III}$

BOX212: maph-1.1(mib12[GFP::maph-1.1]) I; mibIs35 [Prgef-1::BirA $10 \mathrm{ng} / \mu \mathrm{l}$, Pmyo-2::mCherry $2.5 \mathrm{ng} / \mu \mathrm{l}] \mathrm{II}$;

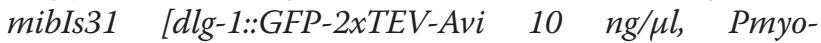
3::mCherry $10 \mathrm{ng} / \mu \mathrm{ll} \mathrm{V}$

SV1311: atad-3(ok3093) II; hels97[Patad-3::ATAD-3, cb unc-119] IV (referred to as ATAD-3 ${ }^{\mathrm{FL}}$ )

SV1312: atad-3(ok3093) II; hels98[atad-3::ATAD-3ETAV, cb unc-119] IV (referred to as ATAD-3 ${ }^{\triangle \mathrm{ETAV}}$ ).

\section{Western blot analysis}

Protein samples were separated on $10 \%$ acrylamide gels, and subjected to western blotting on polyvinylidene difluoride membrane (Immobilon-P; Millipore). Blots were blocked with $5 \%$ skim milk in phosphate-buffered saline with Tween (PBST; $7 \mathrm{mM} \mathrm{Na} 2 \mathrm{HPO}_{4}, 3 \mathrm{mM} \mathrm{NaH} \mathrm{PO}_{4}$, $140 \mathrm{mM} \mathrm{NaCl}, 5 \mathrm{mM} \mathrm{KCl}, 0.05 \%$ Tween-20) for $1 \mathrm{~h}$ at room temperature. For detection of GFP, blots were incubated with rabbit polyclonal anti-GFP (Abcam ab6556, 1:1000) or anti-biotin (Abcam ab1227, 1:1000) in PBST + $5 \%$ skim milk for $1 \mathrm{~h}$ at room temperature, washed with PBST three times for 10 min each at room temperature, incubated with anti-rabbit IgG antibody conjugated to horseradish peroxidase (Jackson Immuno Research $111035003,1: 10,000$ ) for $45 \mathrm{~min}$ at room temperature, washed with PBST three times for 10 min each at room temperature, and finally washed once with PBS at room temperature for $10 \mathrm{~min}$. Blots were developed using enhanced chemiluminescent western blotting substrate (BioRad Laboratories).

\section{C. elegans liquid culture}

Liquid cultures were started with semi-synchronized L1 animals obtained by starvation. Depending on the growth rate of the transgenic strain to be cultured, 20-60 $9 \mathrm{~cm}$ nematode growth media (NGM) plates with OP50 bacteria were seeded with 15-45 L4 animals per plate. After 6 or 7 days at $20^{\circ} \mathrm{C}$, no bacteria were left on the plates and the plates were covered with starved L1 animals. All animals were washed off the plates and transferred to a 21 Erlenmeyer flask containing $500 \mathrm{ml} \mathrm{S}$-medium supplemented with penicillin-streptomycin $(5000 \mathrm{U} / \mathrm{ml}$, Life Technologies 15070-63) diluted 1:100, and nystatin suspension (10,000 U/ml, Sigma N1638) diluted 1:1000 [74]. A pellet of OP50 E. coli bacteria obtained from a $0.5 \mathrm{l}$ overnight culture in lysogeny broth (LB) was added as food source. Animals were allowed to develop until the L3/L4 stage in an incubator at $20^{\circ} \mathrm{C}$ shaking at $200 \mathrm{rpm}$. To harvest the animals, the culture was transferred to $50 \mathrm{ml}$ conical tubes and cooled on ice for $20 \mathrm{~min}$. Animals were then pelleted by centrifugation (all centrifugation steps in this protocol were performed at $400 \mathrm{~g}$ for $2 \mathrm{~min}$ at $4{ }^{\circ} \mathrm{C}$ ). After aspirating the supernatant, animals were pooled in a single $50 \mathrm{ml}$ tube, and washed twice in icecold M9 lacking $\mathrm{MgSO}_{4}$ [74]. After the second wash step, animals were resuspended in $20 \mathrm{ml}$ of ice-cold M9 lacking $\mathrm{MgSO}_{4}$, followed by the addition of $20 \mathrm{ml}$ of ice-cold $60 \%$ sucrose in $\mathrm{H}_{2} \mathrm{O}$. After vigorous mixing of the sucrose/ worm mixture, $4 \mathrm{ml}$ of ice-cold $\mathrm{M} 9$ lacking $\mathrm{MgSO}_{4}$ was gently layered on top, and the worms were centrifuged at $400 \mathrm{~g}$ for $2 \mathrm{~min}$ at $4{ }^{\circ} \mathrm{C}$. A layer of animals was now visible on top of the sucrose, while contaminants sedimented at the bottom. The sucrose float steps were performed as quickly as possible, as otherwise the layer of animals failed to form properly. To maximize recovery, $30 \mathrm{ml}$ of supernatant was aspirated from the sucrose float, and distributed into four $50 \mathrm{ml}$ tubes that were subsequently filled by addition of room-temperature $\mathrm{M} 9$ lacking $\mathrm{MgSO}_{4}$. The room temperature M9 allowed the animals to digest OP50 bacteria in their intestine. The four tubes were placed on ice to cool down for $30 \mathrm{~min}$, after which the animals were washed twice in lysis buffer $(150 \mathrm{mM} \mathrm{NaCl}, 20 \mathrm{mM}$ Tris pH 7.8, 5 mM EDTA). During the first wash the animals were again pooled into one tube. After a final wash in lysis buffer supplemented with $1 \%$ Triton X-100, as much lysis buffer as possible was removed, and the $C$. elegans pellet was frozen in liquid nitrogen and stored at $-80^{\circ} \mathrm{C}$. 


\section{Lysis}

C. elegans pellets were lysed by sonication using a Diagenode Bioruptor (http://www.diagenode.com) fitted with a $15 \mathrm{ml}$ tube holder. To each frozen pellet was added $5 \mathrm{ml}$ of lysis buffer $(150 \mathrm{mM} \mathrm{NaCl}, 20 \mathrm{mM}$ Tris $\mathrm{pH} 7.8$, 5 mM EDTA) supplemented with $1 \%$ Triton X-100, 0.5 tablet of protease inhibitor (Roche, 05892791001), and $7 \mu \mathrm{l}$ of $\beta$-mercaptoethanol. Pellets were gently swirled until thawed. Lysates were transferred to $15 \mathrm{ml}$ TPX hard plastic tubes and placed in the Bioruptor filled with icewater. Samples were lysed nine times for $30 \mathrm{~s}$, with $30 \mathrm{~s}$ intervals. After the third and sixth lysis period, lysates were mixed by gently swirling the tube. To remove cellular debris, lysates were distributed to $2 \mathrm{ml}$ Eppendorf tubes, centrifuged at $16,000 \mathrm{~g}$ for $15 \mathrm{~min}$ at $4{ }^{\circ} \mathrm{C}$, and collected in a fresh conical-bottom $15 \mathrm{ml}$ polypropylene tube. The concentration of protein in the lysates was determined by Bradford assay (Bio-Rad Laboratories) and the lysates were diluted to $1 \mathrm{mg}$ protein/1 ml lysate.

\section{Affinity purification}

Affinity purifications were performed with streptavidincoated beads (Chromotek, HP57.1). Prior to use, beads were washed twice in lysis buffer $(150 \mathrm{mM} \mathrm{NaCl}$, $20 \mathrm{mM}$ Tris $\mathrm{pH}$ 7.8, $5 \mathrm{mM}$ EDTA) supplemented with $1 \%$ Triton X-100 in a $1.5 \mathrm{ml}$ Eppendorf tube, pelleting the beads by centrifugation at $7500 \mathrm{~g}$ for $30 \mathrm{~s}$. After the final wash, beads were resuspended in lysis buffer, in the original volume. Next, $25 \mu \mathrm{l}$ of beads were added to $15 \mathrm{ml}$ of lysate (15 mg protein) in a $15 \mathrm{ml}$ conicalbottom polypropylene tube, after which the tubes were rotated at $4{ }^{\circ} \mathrm{C}$ for $1.5 \mathrm{~h}$. Following the incubation, beads were pelleted by centrifugation at $3220 \mathrm{~g}$ for $5 \mathrm{~min}$ at $4^{\circ}$ $\mathrm{C}$, resuspended in $0.5 \mathrm{ml}$ TEV buffer $(20 \mathrm{mM}$ Tris $\mathrm{pH}$ 8.0, $150 \mathrm{mM} \mathrm{NaCl}$, and $0.3 \% \mathrm{NP} 40$ ), and transferred to an Eppendorf tube. Beads were then washed three times with $500 \mu \mathrm{l}$ TEV buffer, pelleting the beads by centrifugation at $7500 \mathrm{~g}$ for $30 \mathrm{~s}$ at $4{ }^{\circ} \mathrm{C}$. Finally, the beads were resuspended in $15 \mu \mathrm{l} \mathrm{TEV}$ buffer and $2 \mu \mathrm{l}$ of TEV protease (Promega, V6101) was added to the samples. TEV cleavage was performed overnight at $4{ }^{\circ} \mathrm{C}$ in a shaking block for Eppendorf tubes, shaking at $800 \mathrm{rpm}$.

\section{Mass spectrometry}

Protein reduction and alkylation was performed with $10 \mathrm{mM}$ dithiothreitol $\left(56{ }^{\circ} \mathrm{C}\right.$ for $1 \mathrm{~h}$ ) and $50 \mathrm{mM} 2$ chloro-iodoacetamide (30 $\mathrm{min}$ at room temperature in the dark), respectively, after which in-gel digestion was performed with trypsin overnight at $37^{\circ} \mathrm{C}$. Peptides were extracted with $100 \%$ acetonitrile. The samples were analyzed on an LTQ Orbitrap Elite (Thermo Scientific, Bremen) connected to a Proxeon UHPLC system (Thermo Scientific, Odense). The nanoLC was equipped with a
$20 \mathrm{~mm} \times 100 \mu \mathrm{m}$ internal diameter Reprosil C18 trap column and a $400 \mathrm{~mm} \times 50 \mu \mathrm{m}$ internal diameter Poroshell C18 analytical column (Zorbax, Agilent), all packed in-house. Solvent A consisted of $0.1 \mathrm{M}$ acetic acid (Merck) in deionized water (Milli-Q, Millipore), and solvent B consisted of $0.1 \mathrm{M}$ acetic acid in $80 \%$ acetonitrile (Biosolve). Trapping was performed at a flow of $5 \mu \mathrm{l} / \mathrm{min}$ for $10 \mathrm{~min}$ and the fractions were eluted using a flow rate of $150 \mathrm{nl} / \mathrm{min}$ (120 min LC method). The mass spectrometer was operated in positive ion mode and in data-dependent mode to automatically switch between MS and MS/MS. The three most intense ions in the survey scan $(350-1500 \mathrm{~m} / \mathrm{z}$, resolution 60,000 , AGC target 1e6) were fragmented with higher energy collisional dissociation (HCD; AGC target 6e4), with the normalized collision energy set to $32 \%$. The signal threshold for triggering an MS/MS event was set to 500 counts. Charge state screening was enabled, and precursors with unknown charge state or a charge state of 1 were excluded. Dynamic exclusion was enabled (exclusion size list 500, exclusion duration $40 \mathrm{~s}$ ).

\section{Mass spectrometry data analysis}

Peak lists were generated from the raw data files using Proteome Discoverer version 1.4.1.14 (Thermo Scientific, Bremen). For each affinity purification, one peak list was generated per gel lane. Peak lists were searched against a C. elegans database (UniProt, Jan 2014, 25,863 entries) supplemented with frequently observed contaminants using Mascot software version 2.4.01 (Matrix Science, UK). Trypsin was chosen with two missed cleavages allowed. Carbamidomethylation (C) was set as a fixed modification and oxidation $(\mathrm{M})$ was set as variable modification. The searches were performed using a peptide mass tolerance of $50 \mathrm{ppm}$ and a product ion tolerance of $0.05 \mathrm{Da}$ (HCD), followed by data filtering using percolator, resulting in $1 \%$ false discovery rate (FDR). Only ranked 1 peptide spectrum matches with Mascot scores $>20$ were accepted. The spectral counts for each triplicate of controls and the four tissues were uploaded to the CRAPome online interface version 1.1 [42] for statistical validation.

\section{Lysis and immunoprecipitations for DLG-1/ATAD-3}

Strains were grown in S-medium, either containing HB101 bacteria or bacterial feeding strains targeting $d l g$ 1, atad-3, or $g f p$ (control) to induce RNAi. Embryo pellets were obtained by hypochlorite treatment of adult worms. Embryo pellets were ground two times for $30 \mathrm{~s}$ at a frequency of 1500 beats/min using a MikroDismembrator (Sartorius). Ground embryo pellets were lysed in lysis buffer [20 mM Tris- $\mathrm{HCl} \mathrm{pH} \mathrm{7.8,} 250 \mathrm{mM}$ $\mathrm{NaCl}, 15 \%$ glycerol, 1 \% Triton X-100, 0.5 mM EDTA, $1 \mathrm{mM} \beta$-mercaptoethanol, $10 \mathrm{mM}$ 1-naphthyl phosphate 
monosodium salt monohydrate, $50 \mathrm{mM}$ sodium fluoride, $10 \mathrm{mM}$ sodium pyrophosphate decahydrate, $100 \mu \mathrm{M}$ sodium orthovanadate, and protease inhibitors (Roche complete, Mini, EDTA-free)] for $15 \mathrm{~min}$ at $4{ }^{\circ} \mathrm{C}$. The lysate was cleared at $13,000 \mathrm{rpm}$ for $15 \mathrm{~min}$ at $4{ }^{\circ} \mathrm{C}$. For immunoprecipitations, $1 \mathrm{mg}$ of total protein was used with either $1 \mu \mathrm{l}$ mouse anti-PSD95 antibody (Abcam ab2723, RRID AB_303248) non-covalently bound to $5 \mu \mathrm{l}$ protein G Sepharose beads, $1 \mu \mathrm{l}$ rabbit anti-ATAD-3 antibody [44] non-covalently bound to $7.5 \mu \mathrm{l}$ protein A Sepharose beads, $2 \mu \mathrm{l}$ rabbit anti-GFP antibody (ThermoFisher A-11122, RRID AB_10073917) non-covalently bound to $7.5 \mu \mathrm{l}$ protein A Sepharose beads (negative control), or $2 \mu \mathrm{l}$ rabbit anti-eIF4E antibodies [75] noncovalently bound to $7.5 \mu$ l protein A Sepharose beads (negative control). Immunoprecipitations were performed for $1 \mathrm{~h}$ at $4{ }^{\circ} \mathrm{C}$. Input lysates $(1 / 25)$ and immunoprecipitations were loaded on gel. Standard procedures were used for SDS-PAGE and western blotting. Mouse anti-PSD95 (1:1000) and rabbit anti-ATAD-3 (1:500) were used for detection. Horseradish peroxidaseconjugated protein A (VWR International) was used at 1:5000 for ATAD-3 probed blots. The signal was revealed with chemiluminesence (Bio-Rad Laboratories). To examine protein levels in ATAD- $3^{\mathrm{FL}}$, and ATAD$3^{\triangle \mathrm{ETAV}}$ strains, $40 \mathrm{~L} 4$ staged larvae grown at indicated temperatures were collected and boiled for $5 \mathrm{~min}$ in $1 \times$ Laemmli sample buffer. Samples were run on an SDSPAGE gel, and blotted according to standard procedures. Immunoblots were probed with rabbit anti-ATAD-3 (1:500) and mouse anti-actin (1:1000) (MP Biomedicals).

\section{Immunoprecipitations for DLG-1/MAPH-1.1}

Animals were grown and lysed, and GTA-tagged proteins were purified as described above for the MS experiments. Input lysates $(1 / 100)$ and immunoprecipitations were loaded on gel. Standard procedures were used for SDS-PAGE and western blotting. For detection of GFP, blots were incubated with rabbit polyclonal anti-GFP (Abcam ab6556, 1:1000) in PBST $+5 \%$ skim milk for $1 \mathrm{~h}$ at room temperature, washed with PBST three times for $10 \mathrm{~min}$ at room temperature, incubated with antirabbit IgG antibody conjugated to horseradish peroxidase (Jackson Immuno Research 111035003, 1:10,000) for $45 \mathrm{~min}$ at room temperature, washed with PBST three times for $10 \mathrm{~min}$ at room temperature, and finally washed once with PBS at room temperature for $10 \mathrm{~min}$.

\section{Yeast two-hybrid assays}

To generate DB::DLG-1 and DB::BAZ fusions, relative fragments were PCR amplified with primers containing EcoRI and BamHI restriction sites in their tails, and cloned into vector pGBTK7 (Clontech, Palo Alto, CA, USA). The following primers were used: PDZ1_F (5'-
agGAATTCgtcttggagaaaggtcac), PDZ1_R (5'-tggtggGGA TCCcggagccgatgg), PDZ2_F (5' -tccatcgGAATTCattcatcc acc), PDZ2_R (5'-tcccatGGATCCgcggttgtag), PDZ3_F (5'-gactacGAATTCtctcaaatgg), PDZ3_R (5'-atGGATCC ctcttgtggtctgtactg), BAZ_PDZ1-3_F (5'-tgGAATTCgagagc aagcgaaaggagccc), and BAZ_PDZ1-3_R (5'-gcGGATCC caagatcttgcggcctaccagc).

To generate AD::ATAD-3 aa388-595 and AD::ATAD-3 aa388-591 ( $\triangle E T A V)$ fusions, relative fragments were PCR amplified with primers containing BamHI and XhoI restriction sites in their tails, and cloned into vector pACT2 (Clontech). The following primers were used: ATAD-3_F (5'-tcggcGGATCCcaattcataaag), ATAD-3_R595 (5'-taaac CTCGAGttaaacagcagtttctctcttc), and ATAD-3_R591 (5' taaacCTCGAGttatctcttcaacgta). DB and AD plasmids were co-transformed into yeast strain Y190 (Clontech) using the LiAc transformation method [76]. For X-gal assays, yeast were grown overnight at $30{ }^{\circ} \mathrm{C}$ on a nitrocellulose filter on top of a yeast extract peptone dextrose (YEPD) agar plate. A Whatman filter paper was placed in a petri dish containing $2 \mathrm{ml}$ of Z-buffer $(60 \mathrm{mM}$ $\mathrm{Na}_{2} \mathrm{HPO}_{4}, 40 \mathrm{M} \mathrm{NaH} \mathrm{PO}_{4}, 10 \mathrm{M} \mathrm{KCl}, 1 \mathrm{M} \mathrm{MgSO}$, $\mathrm{pH}$ 7), 5.4 $\mu \mathrm{l} \beta$-mercaptoethanol, and 33.4 $\mu \mathrm{l} \mathrm{X-Gal} \mathrm{(stock}$ solution $20 \mathrm{mg} / \mathrm{ml}$ in $\mathrm{N}, \mathrm{N}$-dimethyl formamide). The nitrocellulose filter with yeast was then fixed in liquid nitrogen for $10 \mathrm{~s}$, thawed at room temperature, and placed on the Whatman filter paper for $30 \mathrm{~min}$ at $30^{\circ} \mathrm{C}$.

\section{Generation of atad-3 transgenic strains}

For MosSCI integration of atad-3 constructs on chromosome IV, we generated Patad-3::atad-3::atad-3 3'UTR and Patad-3::atad-3 $E$ ETAV::atad-3 3'UTR constructs, and cloned these into the pCFJ1178 vector (a gift from E. Jorgensen, HHMI, University of Utah, USA). For Patad-3 we used a 1050 bp 5' region of atad-3. As a 3' UTR we used a 300 bp flanking region of atad-3. Patad3::atad-3-ETAV::atad-3 3'UTR lacked 12 bp at the 3'end of the coding region. MosSCI integration was performed as previously described [46, 77]. Briefly: the following injection mixture was injected into unc-119 animals: $10 \mathrm{ng} / \mu \mathrm{l}$ targeting vector, $10 \mathrm{ng} / \mu \mathrm{l} \mathrm{pCFJ601}$ (Peft-3:Mos1 transposase), $2.5 \mathrm{ng} / \mu \mathrm{l}$ pCFJ90 (Pmyo2:mCherry:unc-54 UTR), and $10 \mathrm{ng} / \mu \mathrm{l}$ pGH8 (Prab3:mCherry:unc-54 UTR), and wild-type non-fluorescent animals were selected from the F2 progeny.

\section{Progeny counting and scoring of embryonic lethality}

Starting at the L4 stage, individual animals were cultured at $15{ }^{\circ} \mathrm{C}$ or $25^{\circ} \mathrm{C}$ and transferred to a fresh plate every $24 \mathrm{~h}$. Hatched and unhatched progeny were counted $24 \mathrm{~h}$ after removal of the P0. Bars in Fig. 6 represent mean values, and error bars the standard deviation. The progeny of four animals was counted for each genotype and temperature. 


\section{Phylogenetic analysis and protein alignments}

To identify proteins related to MAPH-1.1, MAPH-1.2, and MAPH-1.3, we performed an iterative JackHMMER search with each of the three proteins against the Reference Proteomes dataset (three iterations). To generate the phylogenetic tree, we selected MAP1 homologs from a subset of species. These sequences were aligned using the online version of MAFFT with the settings E-INS-i iterative refinement method (http://mafft.cbrc.jp/alignment/server/) [78]. From the aligned sequences, a phylogenetic tree was produced using FastTree 2 with the default settings [79]. The online Interactive Tree of Life tool was used to visualize the phylogenetic tree (http:// itol.embl.de/) [80]. To calculate similarity of MAPH-1.1 to human MAP1S and Drosophila Futsch, we performed pairwise alignments with MAFFT as described above, and calculated protein similarity using the online Sequence Manipulation Suite (http://www.bioinformatics.org/sms2/ident_sim.html) [81].

\section{Genome engineering of GFP::maph-1.1}

To engineer the GFP::maph-1 locus, we used homology directed repair of a CRISPR/Cas9 induced double strand break (DSB). To increase the efficiency of DSB generation we generated a new subgenomic RNA (sgRNA) expression vector (pJJR50) that contains the A-U flipped and hairpin extended sgRNA sequence described in Chen et al. [82], under control of the R07E5.16 U6 promoter [83]. Target sequences are cloned into BbsI digested vector as pairs of annealed oligonucleotides with a $5^{\prime}$-TCTT overhang added to the forward oligo, and a $5{ }^{\prime}$-AAAC overhang added to the reverse oligo. The maph-1.1 sgRNA target sequence was GCGTGCATCTACGTCTTGGG, and oligos used to insert the sequence into pJJR50 were 5'tcttGCGTGCATCTACGTCTTGGG and 5'- aacCCCAAGACGTAGATGCACGC. To generate the repair template, we inserted $450-650$ bp sequences flanking the start codon of maph-1.1 into the self-excising selection cassette vector pDD282 as described [84]. Several mutations were introduced into the sgRNA target site to prevent cutting by Cas 9 after repair. The following mixture was injected into $20 \mathrm{~N} 2$ adults: $50 \mathrm{ng} / \mathrm{ml}$ Peft-3::Cas9 (Addgene \#46168) [85], $100 \mathrm{ng} / \mathrm{ml} \mathrm{sgRNA} \mathrm{in} \mathrm{pJJR50,}$ $20 \mathrm{ng} / \mathrm{ml}$ maph-1.1 repair template, and $2.5 \mathrm{ng} / \mathrm{ml} \mathrm{pCFJ90}$ [46]. Injected animals were placed on individual NGM plates. After $2-3$ days at $25^{\circ} \mathrm{C}, 500 \mu \mathrm{l}$ of $5 \mathrm{mg} / \mathrm{ml}$ hygromycin in water was added to each plate, and healthy nonred-fluorescent Rol animals were selected after 3-5 days. To eliminate the marker cassette, 8-16 L2 animal were heat shocked for $4 \mathrm{~h}$ at $34{ }^{\circ} \mathrm{C}$, and non-Rol progeny were selected. We obtained two independent lines, both of which displayed the same expression pattern. Vector maps and sequences are available in Additional file 16.
Expression in hippocampal neurons and fixation

To express MAPH-1.1 and DLG-1 in hippocampal neurons, we generated constructs consisting of MAPH1.1 N-terminally tagged with GFP, and DLG-1 Cterminally tagged with mCherry, driven by the $\beta$ actin promoter. MAPH-1.1 and DLG-1 were PCR amplified from a mixed-stage C. elegans cDNA library, and inserted into a vector containing the $\mathrm{p} \beta$ actin promoter and GFP or mCherry [86]. MAPH-1.1 was amplified using primers 5'-aaaGGCGCGCCAatgccggaggaatatatc atg and $5^{\prime}$-tttGCGGCCGCttagagcaaatcgactctggcc, and cloned using AscI and NotI. DLG-1 was amplified using primers 5'-aaaAAGCTTatgtcccacgagtcatcgg and 5'tttGGTCTCGTCGACttatgacgtggcacccaaattggcg,

digested with HindIII and BsaI, and ligated into vector digested with HindIII and SalI. Vector maps and sequences are available in Additional file 16. Primary hippocampal neurons prepared from embryonic day 18 rat brains [86] were transfected at 4 days in vitro (DIV4) for MAPH-1 and microtubule imaging, or DIV22 for DLG-1 imaging, using Lipofectamine 2000 (Life Technologies) and cultured for 2 additional days. For microtubule imaging, cells were first extracted with $0.3 \%$ glutaraldehyde (GA) in PEM80-buffer (80 mM PIPES, 1 mM EGTA, $4 \mathrm{mM} \mathrm{MgCl} 2, \mathrm{pH}$ 6.9) for $1 \mathrm{~min}$ at $37^{\circ} \mathrm{C}$. Next, fixation was performed in $4 \%$ paraformaldehyde (PFA) for $10 \mathrm{~min}$. Subsequently, cells were washed twice for $5 \mathrm{~min}$ in PBS (Lonza BE17-517Q, w/o Ca and Mg) and cells were further permeabilized for 10 min in PBS $+0.2 \%$ TritonX100. Cells were then washed three times for $5 \mathrm{~min}$ in PBS and incubated for $45 \mathrm{~min}$ in blocking solution ( $2 \% \mathrm{w} / \mathrm{v}$ bovine serum albumin [BSA], $0.2 \% \mathrm{w} / \mathrm{v}$ gelatin, $10 \mathrm{mM}$ glycine, $50 \mathrm{mM} \mathrm{NH}_{4} \mathrm{Cl}$ in PBS, $\mathrm{pH}$ 7.4). Primary antibodies to $\alpha$-tubulin (Sigma, mouse clone B-5-1-2, 1:800) and MAP2 (Abcam ab5392, chicken, 1:1000) were incubated overnight at $4{ }^{\circ} \mathrm{C}$ in blocking solution. Cells were washed three times in PBS and incubated with secondary antibodies anti-Mouse Alexa Fluor568 (Life Technologies, goat, 1:800) and anti-Chicken Alexa Fluor647 (Life Technologies, goat, 1:800) for $1.5 \mathrm{~h}$ at room temperature. Finally, cells were washed three times in PBS and mounted in Mowiol 4-88 (Sigma-Aldrich). For imaging of postsynaptic densities, fixation (without prior extraction) was performed in $4 \%$ PFA for $10 \mathrm{~min}$. Subsequently, cells were washed three times for $10 \mathrm{~min}$ in PBS (Lonza BE17-517Q, w/o Ca and Mg), and mounted in Mowiol 4-88.

\section{Microscopy and image processing}

Still images of live animals were captured on a spinning disc platform consisting of a Nikon Ti-U inverted microscope with a motorized XY stage and a Piezo $\mathrm{Z}$ stage, $\times 60$ and $\times 100$ PLAN APO 1.4 numerical aperture (NA) oil objectives, a Yokogawa CSU-X1 spinning disc unit equipped 
with a dual dichroic mirror set for laser wavelengths $488 \mathrm{~nm}$ and $561 \mathrm{~nm}, 488 \mathrm{~nm}$ and $561 \mathrm{~nm}$ solid state $50 \mathrm{~mW}$ lasers controlled by an Andor revolution 500 series AOTF Laser modulator and combiner, Semrock 512/23+ 630/91 dual band pass emission filter, Semrock 525/30 single band pass emission filter, Semrock 617/73 single band pass filter, Semrock 4800 long pass filter (500-1200 pass), and an Andor iXON DU-885 monochrome EMCCD+ camera. All components were controlled by MetaMorph Microscopy Automation \& Image Analysis Software. Live imaging was performed on a Nikon Eclipse-Ti microscope with a Plan Apo VC, ×60, 1.40 NA oil objective (Nikon). The microscope was equipped with an ASI motorized stage MS-2000-XYZ with Piezo Top Plate and a Perfect Focus System (Nikon), and used MetaMorph 7.8 to control the camera and all motorized parts. Confocal excitation and detection was achieved using a $100 \mathrm{~mW}$ Cobolt Calypso laser, Yokogawa spinning disc confocal scanning unit (CSU-X1A1), a GFP emission filter [ET-GFP (49002); Chroma], and a Photometrics Evolve 512 EMCCD camera at a final magnification of $110 \mathrm{~nm}$ per pixel, including an additional magnification introduced by an extra intermediate lens 2.0X (Edmund Optics). For FRAP experiments we used a similar microscope setup equipped with an ILas system (Roper Scientific France/PICT-IBiSA, Institut Curie), with the laser set at $100 \%$ laser power and a Plan Apo $\times 60$ NA 1.40 oil lens. Imaging was performed at 1 or 2 frames per second. Microscopy of fixed samples was performed on a Zeiss LSM700 laser scanning confocal microscope equipped with a $\times 63$ Plan-Apochromat 1.4 NA objective; $405 \mathrm{~nm}, 488 \mathrm{~nm}, 555 \mathrm{~nm}$, and $633 \mathrm{~nm}$ lasers; and the following emission filters: SP490 (400-490 nm), SP555 (400-555 nm), SP640 (400-640 nm), BP490-555 (490$555 \mathrm{~nm})$, LP560 (560-750 nm), LP640 (640-750 nm), and BP592-662 (592-662 nm). The LSM700 was controlled by the Zen software package. Maximum projections were generated from a series of slices of a Z-stack with ImageJ and processed with Adobe Photoshop CS6 and Adobe Illustrator CS6.

\section{FRAP analysis}

Images were processed and analyzed in ImageJ. For the FRAP analysis, the average gray value of a $100 \times 10$ pixel region in the FRAP region or a similar non-bleach area was calculated and background subtracted frame-byframe by subtracting the average intensity of an empty, non-bleached area. FRAP recovery was calculated as the recovery from the first frame after bleach (set to 0 ) normalized to the average of the five frames before bleach.

\section{Microtubule depolymerization speed measurement}

Microtubule depolymerization speeds were calculated by making kymographs of the acquired movies using the KymoResliceWide plugin in ImageJ and measuring the distance and time of a depolymerization event. Graphs in Additional file 15: Figure S6 show the mean \pm standard deviation, as well as individual measurements.

\section{Additional files}

\begin{abstract}
Additional file 1: Figure S1. C. elegans optimized BirA. a DNA sequence of BirA, codon optimized for C. elegans and including four artificial introns, as well as a Myc tag. $\mathbf{b}$ Analysis of the expression and splicing of BirA expressed from the neuronal rgef-1 promoter by RT-PCR. Primer locations relative to the BirA coding sequence are indicated. Each RT-PCR results in a band with the expected size for a properly expressed and spliced BirA cDNA. (PDF $195 \mathrm{~kb}$ )
\end{abstract}

Additional file 2: Figure S2. DNA sequence of the C-terminal GTA tag. Relevant regions are highlighted in color. (PDF $88 \mathrm{~kb}$ )

Additional file 3: Figure S3. DNA sequence of the N-terminal GTA tag. Relevant regions are highlighted in color. (PDF $88 \mathrm{~kb}$ )

Additional file 4: Table S1. All proteins identified, organized by tissue examined. (XLSX $118 \mathrm{~kb})$

Additional file 5: Table S2. All proteins identified, organized by bait protein. (XLSX $140 \mathrm{~kb}$ )

Additional file 6: Table S3. Expanded copy of Table 2 in the main text including peptide numbers. (XLSX $14 \mathrm{~kb}$ )

Additional file 7: Figure S4. ATAD- $3^{\mathrm{FL}}$ and $A T A D^{\Delta E T A V}$ are expressed at similar levels. Western blot analysis showing expression levels of ATAD-3 in ATAD-3 $3^{\mathrm{FL}}$ and $A T A D^{\triangle \mathrm{ETAV}}$ transgenic animals at three different temperatures. (PDF $244 \mathrm{~kb}$ )

Additional file 8: Figure S5. a Phylogenetic tree of MAP1-related proteins. Proteins were identified through iterative HMMER searches as implemented in JackHMMER. Three major groups containing the human MAP1A, MAP1B, and MAP1S proteins are color coded. b Sequence alignment of MAPH-1.1 with human MAP1A. (PDF $231 \mathrm{~kb}$ )

Additional file 9: Movie S1. Live imaging of GFP::MAPH-1.1 in body wall muscle cells. (MP4 $4269 \mathrm{~kb}$ )

Additional file 10: Movie S2. Live imaging of GFP::MAPH-1.1 in the hypodermis. (MP4 $2275 \mathrm{~kb})$

Additional file 11: Movie S3. Live imaging of GFP::MAPH-1.1 in head region showing nerve ring. (MP4 $2266 \mathrm{~kb}$ )

Additional file 12: Movie S4. Live imaging of GFP::MAPH-1.1 in posterior pharynx bulb. (MP4 $2252 \mathrm{~kb}$ )

Additional file 13: Movie S5. Live imaging of GFP::MAPH-1.1 in seam cells. (MP4 $2276 \mathrm{~kb}$ )

Additional file 14: Movie S6. Example of FRAP analysis in hypodermis. (MP4 3024 kb)

Additional file 15: Figure S6: GFP::MAPH-1 dynamics. a Fluorescence recovery of GFP::MAPH-1.1 after photobleaching in the hypodermis. Shown is the mean \pm SEM of seven measurements. Dotted horizontal line indicates the maximum recovery (average of the final 10 time points, 0.724). Dotted vertical line indicates the time point (6 s) of $50 \%$ recovery of the maximum. See Additional file 14: Movie S6 for an example. b Microtubule depolymerization rates in the hypodermis (Hyp) and body wall muscle (BWM). Shown is the mean $\pm S D$, as well as individual measurements. Hyp $n=14$, BWM $n=18$. (PDF $139 \mathrm{~kb}$ )

Additional file 16: PDF document with all vector maps and sequences. (PDF $1724 \mathrm{~kb}$ )

\section{Acknowledgements}

Some strains were provided by the CGC, which is funded by the NIH Office of Research Infrastructure Programs (P40 OD010440). We thank WormBase for curating and making available data related to C. elegans, Oliver Hobert for providing the original DNA of the optimized BirA, and Suzan Ruijtenberg for help with injections. This work was supported by the Netherlands Organization for Scientific Research (NWO)/ALW Innovational Research Incentives Scheme Vidi grant 864.09.008 to MB, NWO/CW ECHO grant 711.014.005 to MB, NWO/ 
ALW Veni grant 863.12.001 to MH, and NWO/CW ECHO grant 711.011.010 to SvdH. TYL, SSG, ADZ, and JM have been supported by the Netherlands Proteomics Centre, the NWO supporting the Roadmap embedded large-scale proteomics facility Proteins@Work (project 184.032.201), and by the PRIME-XS project grant agreement number 262067 supported by the European Community's Seventh Framework Programme (FP7/2007-2013) to AJRH. OB was supported by grants BO1061/9-1 and BO1061/11-3 from the DFG. BT has been supported by the Marie Curie Re-integration Grant (CIG PCIG12-GA-2012333922) of the European Community's Seventh Framework Programme and the ERC Starting Grant (REPROWORM_637530) of the European Community's Horizon 2020 Framework Programme.

\section{Availability of data and materials}

The MS proteomics data have been deposited to the ProteomeXchange Consortium [87] via the PRIDE partner repository with the dataset identifier PXD002139. The following plasmids are available from Addgene: pMB37 (\#73084), pMB41 (\#73087), pMB71 (\#73085), pMB72 (\#73088), pMB73 (\#73086), pBT331 (\#79971)

\section{Authors' contributions}

BT and MB conceived the tissue-specific purification approach. MB supervised its implementation. SW executed the majority of experiments. JM, SSG, TYL, and ADZ-D performed the mass spectrometry experiments and data analysis. CB performed the ATAD-3 experiments. MH performed the yeast two-hybrid analysis. TK and RPT performed the mammalian MAPH-1 experiments. JJR generated and analyzed the GFP::MAPH-1.1 strains. MH performed the live imaging, FRAP analysis, and microtubule depolymerization measurements. SS performed the BirA splicing analysis. JK provided technical assistance. $\mathrm{CCH}, \mathrm{OB}, \mathrm{BT}$, SvdH, and AJRH, provided co-supervision, technical expertise, and helpful discussions in the project. SW and MB wrote the manuscript. All authors read and approved the final manuscript.

\section{Competing interests}

The authors declare that they have no competing interests.

\section{Author details}

'Developmental Biology, Department of Biology, Faculty of Science, Utrecht University, Padualaan 8, $3584 \mathrm{CH}$ Utrecht, The Netherlands. ${ }^{2}$ Biomolecular Mass Spectrometry and Proteomics, Bijvoet Center for Biomolecular Research and Utrecht Institute for Pharmaceutical Sciences, Utrecht University, Padualaan 8, $3584 \mathrm{CH}$ Utrecht, The Netherlands. ${ }^{3}$ Netherlands Proteomics Centre, Padualaan 8, $3584 \mathrm{CH}$ Utrecht, The Netherlands. ${ }^{4}$ Institut für Wissenschaftliche Medizin, D-40591 Düsseldorf, Germany. ${ }^{5}$ Cell Biology, Department of Biology, Faculty of Science, Utrecht University, Padualaan 8, $3584 \mathrm{CH}$ Utrecht, The Netherlands. ${ }^{6}$ Berlin Institute for Medical Systems Biology (BIMSB), Max Delbrueck Center for Molecular Medicine (MDC) in the Helmholtz Association, Robert-Roessle-Strasse 10, Berlin 13125, Germany. ${ }^{7}$ Molecular Cell Biology, Anatomy I, University of Cologne, D-50937 Cologne, Germany. ${ }^{8}$ Present address: Department of Physiology, Radboud University Medical Center, Geert Grooteplein 26, 6525 GA Nijmegen, The Netherlands. ${ }^{9}$ Present address: Proteomics Unit, Spanish National Cancer Research Centre (CNIO), ProteoRed-ISCIII, 28029 Madrid, Spain. ${ }^{10}$ Present address: Center for Cancer Research and Department of Pathology, Massachusetts General Hospital and Harvard Medical School Department of Pathology, 149 13th Street, 02129 Charlestown, MA, USA.

Received: 8 June 2016 Accepted: 19 July 2016

Published online: 09 August 2016

\section{References}

1. Altelaar AFM, Munoz J, Heck AJR. Next-generation proteomics: towards an integrative view of proteome dynamics. Nat Rev Genet. 2013;14:35-48.

2. Butland G, Peregrín-Alvarez JM, Li J, Yang W, Yang X, Canadien V, et al. Interaction network containing conserved and essential protein complexes in Escherichia coli. Nature. 2005;433:531-7.

3. Gavin A-C, Aloy P, Grandi P, Krause R, Boesche M, Marzioch M, et al. Proteome survey reveals modularity of the yeast cell machinery. Nature. 2006:440:631-6.

4. Guruharsha KG, Rual J-F, Zhai B, Mintseris J, Vaidya P, Vaidya N, et al. A protein complex network of Drosophila melanogaster. Cell. 2011;147: 690-703.
5. Ho Y, Gruhler A, Heilbut A, Bader GD, Moore L, Adams S-L, et al. Systematic identification of protein complexes in Saccharomyces cerevisiae by mass spectrometry. Nature. 2002;415:180-3.

6. Hu P, Janga SC, Babu M, Díaz-Mejía JJ, Butland G, Yang W, et al. Global functional atlas of Escherichia coli encompassing previously uncharacterized proteins. PLoS Biol. 2009;7, e96.

7. Krogan NJ, Cagney G, Yu H, Zhong G, Guo X, lgnatchenko A, et al. Global landscape of protein complexes in the yeast Saccharomyces cerevisiae. Nature. 2006:440:637-43.

8. Kühner S, van Noort V, Betts MJ, Leo-Macias A, Batisse C, Rode M, et al. Proteome organization in a genome-reduced bacterium. Science. 2009;326:1235-40.

9. Ori A, Iskar M, Buczak K, Kastritis P, Parca L, Andrés-Pons A, et al. Spatiotemporal variation of mammalian protein complex stoichiometries. Genome Biol. 2016;17:47.

10. Cheeseman IM, Niessen S, Anderson S, Hyndman F, Yates JR, Oegema K, et al. A conserved protein network controls assembly of the outer kinetochore and its ability to sustain tension. Genes Dev. 2004;18:2255-68.

11. Desai A, Rybina S, Müller-Reichert T, Shevchenko A, Shevchenko A, Hyman $A$, et al. KNL-1 directs assembly of the microtubule-binding interface of the kinetochore in C. elegans. Genes Dev. 2003;17:2421-35.

12. Ding $L$, Spencer A, Morita $K$, Han M. The developmental timing regulator AIN-1 interacts with miRISCs and may target the argonaute protein ALG-1 to cytoplasmic P bodies in C. elegans. Mol Cell. 2005;19:437-47.

13. Duchaine TF, Wohlschlegel JA, Kennedy S, Bei Y, Conte D, Pang K, et al. Functional proteomics reveals the biochemical niche of $C$. elegans DCR-1 in multiple small-RNA-mediated pathways. Cell. 2006;124:343-54.

14. Gassmann R, Essex A, Hu J-S, Maddox PS, Motegi F, Sugimoto A, et al. A new mechanism controlling kinetochore-microtubule interactions revealed by comparison of two dynein-targeting components: SPDL-1 and the Rod/ Zwilch/Zw10 complex. Genes Dev. 2008;22:2385-99.

15. Gottschalk A, Almedom RB, Schedletzky T, Anderson SD, Yates JR, Schafer WR. Identification and characterization of novel nicotinic receptorassociated proteins in Caenorhabditis elegans. EMBO J. 2005;24:2566-78.

16. Gu W, Shirayama M, Conte D, Vasale J, Batista PJ, Claycomb JM, et al. Distinct argonaute-mediated 22G-RNA pathways direct genome surveillance in the C. elegans germline. Mol Cell. 2009;36:231-44.

17. Moresco JJ, Carvalho PC, Yates JR. Identifying components of protein complexes in C. elegans using co-immunoprecipitation and mass spectrometry. J Proteomics. 2010;73:2198-204.

18. Polanowska J, Martin JS, Garcia-Muse T, Petalcorin MIR, Boulton SJ. A conserved pathway to activate BRCA1-dependent ubiquitylation at DNA damage sites. EMBO J. 2006;25:2178-88.

19. Schäffer U, Schlosser A, Müller KM, Schäfer A, Katava N, Baumeister R, et al. SnAvi-a new tandem tag for high-affinity protein-complex purification. Nucleic Acids Res. 2010;38, e91.

20. Srinivasan DG, Fisk RM, Xu H, van den Heuvel S. A complex of LIN-5 and GPR proteins regulates $G$ protein signaling and spindle function in $C$ elegans. Genes Dev. 2003;17:1225-39.

21. van der Voet M, Berends CWH, Perreault A, Nguyen-Ngoc T, Gönczy P, Vidal M, et al. NuMA-related LIN-5, ASPM-1, calmodulin and dynein promote meiotic spindle rotation independently of cortical LIN-5/GPR/Galpha. Nat Cell Biol. 2009;11:269-77.

22. Zhang $L$, Ding $L$, Cheung $T H$, Dong $M-Q$, Chen J, Sewell AK, et al. Systematic identification of $C$. elegans miRISC proteins, miRNAs, and mRNA targets by their interactions with GW182 proteins AIN-1 and AIN-2. Mol Cell. 2007;28:598-613.

23. de Boer E, Rodriguez P, Bonte E, Krijgsveld J, Katsantoni E, Heck A, et al. Efficient biotinylation and single-step purification of tagged transcription factors in mammalian cells and transgenic mice. Proc Natl Acad Sci U S A. 2003;100:7480-5.

24. Schatz PJ. Use of peptide libraries to map the substrate specificity of a peptide-modifying enzyme: a 13 residue consensus peptide specifies biotinylation in Escherichia coli. Biotechnology. 1993;11:1138-43.

25. Aspöck G, Kagoshima H, Niklaus G, Bürglin TR. Caenorhabditis elegans has scores of hedgehog-related genes: sequence and expression analysis. Genome Res. 1999:9:909-23.

26. Tursun B, Cochella L, Carrera I, Hobert O. A toolkit and robust pipeline for the generation of fosmid-based reporter genes in C. elegans. PLoS One. 2009; 4, e4625.

27. Christner JE, Schlesinger MJ, Coon MJ. Enzymatic activation of biotin. Biotinyl adenylate formation. J Biol Chem. 1964;239:3997-4005. 
28. Aceto D, Beers M, Kemphues KJ. Interaction of PAR- 6 with CDC-42 is required for maintenance but not establishment of PAR asymmetry in C. elegans. Dev Biol. 2006;299:386-97.

29. Betschinger J, Mechtler K, Knoblich JA. The Par complex directs asymmetric cell division by phosphorylating the cytoskeletal protein Lgl. Nature. 2003; 422:326-30.

30. Izumi Y, Hirose T, Tamai Y, Hirai S, Nagashima Y, Fujimoto T, et al. An atypical PKC directly associates and colocalizes at the epithelial tight junction with ASIP, a mammalian homologue of Caenorhabditis elegans polarity protein PAR-3. J Cell Biol. 1998;143:95-106.

31. Lockwood CA, Lynch AM, Hardin J. Dynamic analysis identifies novel roles for DLG-1 subdomains in AJM-1 recruitment and LET-413-dependent apical focusing. J Cell Sci. 2008;121:1477-87.

32. Plant PJ, Fawcett JP, Lin DCC, Holdorf AD, Binns K, Kulkarni S, et al. A polarity complex of mPar-6 and atypical PKC binds, phosphorylates and regulates mammalian Lgl. Nat Cell Biol. 2003;5:301-8.

33. Yamanaka T, Horikoshi Y, Sugiyama Y, Ishiyama C, Suzuki A, Hirose T, et al. Mammalian Lgl forms a protein complex with PAR-6 and aPKC independently of PAR-3 to regulate epithelial cell polarity. Curr Biol. 2003;13:734-43

34. Palmer E, Freeman T. Investigation into the use of C- and N-terminal GFP fusion proteins for subcellular localization studies using reverse transfection microarrays. Comp Funct Genomics. 2004;5:342-53.

35. Stathakis DG, Hoover KB, You Z, Bryant PJ. Human postsynaptic density-95 (PSD95): location of the gene (DLG4) and possible function in nonneural as well as in neural tissues. Genomics. 1997:44:71-82.

36. Gotta M, Abraham MC, Ahringer J. CDC-42 controls early cell polarity and spindle orientation in C. elegans. Curr Biol. 2001;11:482-8.

37. Kemphues KJ, Priess JR, Morton DG, Cheng NS. Identification of genes required for cytoplasmic localization in early C. elegans embryos. Cell. 1988;52:311-20

38. Beatty A, Morton D, Kemphues K. The C. elegans homolog of Drosophila Lethal giant larvae functions redundantly with PAR-2 to maintain polarity in the early embryo. Development. 2010;137:3995-4004.

39. Hoege C, Constantinescu A-T, Schwager A, Goehring NW, Kumar P, Hyman AA. LGL can partition the cortex of one-cell Caenorhabditis elegans embryos into two domains. Curr Biol. 2010;20:1296-303.

40. Legouis R, Gansmuller A, Sookhareea S, Bosher JM, Baillie DL, Labouesse M. LET-413 is a basolateral protein required for the assembly of adherens junctions in Caenorhabditis elegans. Nat Cell Biol. 2000;2:415-22.

41. Choi H, Larsen B, Lin Z-Y, Breitkreutz A, Mellacheruvu D, Fermin D, et al. SAINT: probabilistic scoring of affinity purification-mass spectrometry data Nat Methods. 2011:8:70-3.

42. Mellacheruvu D, Wright Z, Couzens AL, Lambert J-P, St-Denis NA, Li T, et al. The CRAPome: a contaminant repository for affinity purification-mass spectrometry data. Nat Methods. 2013;10:730-6.

43. Yap SF, Chen W, Lim L. Molecular characterization of the Caenorhabditis elegans Rho GDP-dissociation inhibitor. Eur J Biochem FEBS. 1999;266:1090-100.

44. Hoffmann M, Bellance N, Rossignol R, Koopman WJH, Willems PHGM, Mayatepek E, et al. C. elegans ATAD-3 is essential for mitochondrial activity and development. PLoS One. 2009;4, e7644.

45. Tonikian R, Zhang Y, Sazinsky SL, Currell B, Yeh J-H, Reva B, et al. A specificity map for the PDZ domain family. PLoS Biol. 2008;6, e239.

46. Frøkjær-Jensen C, Davis MW, Ailion M, Jorgensen EM. Improved Mos1mediated transgenesis in C. elegans. Nat Methods. 2012;9:117-8.

47. Hummel T, Krukkert K, Roos J, Davis G, Klämbt C. Drosophila Futsch/22C10 is a MAP1B-like protein required for dendritic and axonal development. Neuron. 2000:26:357-70.

48. Brenman JE, Topinka JR, Cooper EC, McGee AW, Rosen J, Milroy T, et al. Localization of postsynaptic density-93 to dendritic microtubules and interaction with microtubule-associated protein 1A. J Neurosci. 1998;18:8805-13.

49. Reese ML, Dakoji S, Bredt DS, Dötsch V. The guanylate kinase domain of the MAGUK PSD-95 binds dynamically to a conserved motif in MAP1a. Nat Struct Mol Biol. 2007;14:155-63

50. Powell S, Forslund K, Szklarczyk D, Trachana K, Roth A, Huerta-Cepas J, et al. eggNOG v4.0: nested orthology inference across 3686 organisms. Nucleic Acids Res. 2014;42:D231-9.

51. Lacroix B, Bourdages KG, Dorn JF, Ihara S, Sherwood DR, Maddox PS, et al. In situ imaging in C. elegans reveals developmental regulation of microtubule dynamics. Dev Cell. 2014;29:203-16.
52. Wang S, Wu D, Quintin S, Green RA, Cheerambathur DK, Ochoa SD, et al. NOCA-1 Functions with $\gamma$-tubulin and in parallel to Patronin to assemble noncentrosomal microtubule arrays in C. elegans. eLife. 2015;4:e08649.

53. Richardson CE, Spilker KA, Cueva JG, Perrino J, Goodman MB, Shen K. PTRN-1, a microtubule minus end-binding CAMSAP homolog, promotes microtubule function in Caenorhabditis elegans neurons. eLife. 2014;3, e01498.

54. Bulinski JC, Odde DJ, Howell BJ, Salmon TD, Waterman-Storer CM. Rapid dynamics of the microtubule binding of ensconsin in vivo. J Cell Sci. 2001; 114:3885-97.

55. Halpain S, Dehmelt L. The MAP1 family of microtubule-associated proteins. Genome Biol. 2006;7:224

56. Kallay LM, McNickle A, Brennwald PJ, Hubbard AL, Braiterman LT. Scribble associates with two polarity proteins, Lgl2 and Vangl2, via distinct molecular domains. J Cell Biochem. 2006;99:647-64.

57. Ooi SL, Henikoff JG, Henikoff S. A native chromatin purification system for epigenomic profiling in Caenorhabditis elegans. Nucleic Acids Res. 2010;38, e26.

58. Steiner FA, Talbert PB, Kasinathan S, Deal RB, Henikoff S. Cell-type-specific nuclei purification from whole animals for genome-wide expression and chromatin profiling. Genome Res. 2012;22:766-77.

59. Li B, Kim H, Beers M, Kemphues K. Different domains of $C$. elegans PAR-3 are required at different times in development. Dev Biol. 2010;344:745-57.

60. Fang $\mathrm{H}-\mathrm{Y}$, Chang $\mathrm{C}-\mathrm{L}$, Hsu S-H, Huang $\mathrm{C}-\mathrm{Y}$, Chiang S-F, Chiou S-H, et al. ATPase family AAA domain-containing $3 \mathrm{~A}$ is a novel anti-apoptotic factor in lung adenocarcinoma cells. J Cell Sci. 2010;123:1171-80.

61. Gilquin B, Taillebourg E, Cherradi N, Hubstenberger A, Gay O, Merle N, et al. The AAA+ ATPase ATAD3A controls mitochondrial dynamics at the interface of the inner and outer membranes. Mol Cell Biol. 2010;30:1984-96.

62. Hubstenberger A, Labourdette G, Baudier J, Rousseau D. ATAD 3A and ATAD 3B are distal 1p-located genes differentially expressed in human glioma cell lines and present in vitro anti-oncogenic and chemoresistant properties. Exp Cell Res. 2008;314:2870-83.

63. Schaffrik M, Mack B, Matthias C, Rauch J, Gires O. Molecular characterization of the tumor-associated antigen AAA-TOB3. Cell Mol Life Sci. 2006;63:2162-74.

64. Bogenhagen DF, Rousseau D, Burke $S$. The layered structure of human mitochondrial DNA nucleoids. J Biol Chem. 2008;283:3665-75.

65. Hubstenberger A, Merle N, Charton R, Brandolin G, Rousseau D. Topological analysis of ATAD3A insertion in purified human mitochondria. J Bioenerg Biomembr. 2010:42:143-50.

66. Chiang S-F, Huang C-Y, Lin T-Y, Chiou S-H, Chow K-C. An alternative import pathway of AlF to the mitochondria. Int J Mol Med. 2012;29:365-72.

67. Geuijen CAW, Bijl N, Smit RCM, Cox F, Throsby M, Visser TJ, et al. A proteomic approach to tumour target identification using phage display, affinity purification and mass spectrometry. Eur J Cancer. 2005:41:178-87.

68. Ben-Menachem R, Tal M, Shadur T, Pines O. A third of the yeast mitochondrial proteome is dual localized: a question of evolution. Proteomics. 2011;11:4468-76.

69. Firestein BL, Rongo C. DLG-1 is a MAGUK similar to SAP97 and is required for adherens junction formation. Mol Biol Cell. 2001;12:3465-75.

70. Zou B, Yan H, Kawasaki F, Ordway RW. MAP1 structural organization in Drosophila: in vivo analysis of FUTSCH reveals heavy- and light-chain subunits generated by proteolytic processing at a conserved cleavage site. Biochem J. 2008;414:63-71

71. Manil-Ségalen M, Lefebvre $C$, Jenzer C, Trichet M, Boulogne C, SatiatJeunemaitre $B$, et al. The $C$. elegans LC3 acts downstream of GABARAP to degrade autophagosomes by interacting with the HOPS subunit VPS39. Dev Cell. 2014:28:43-55.

72. Waaijers S, Boxem M. Engineering the Caenorhabditis elegans genome with CRISPR/Cas9. Methods. 2014;68:381-8.

73. Brenner S. The genetics of Caenorhabditis elegans. Genetics. 1974;77:71-94.

74. Wood WB. The Community of C. elegans Researchers. The Nematode Caenorhabditis elegans. Cold Spring Harbor Laboratory: Cold Spring Harbor; 1988.

75. Kleijn M, Voorma HO, Thomas AA. Phosphorylation of elF-4E and initiation of protein synthesis in P19 embryonal carcinoma cells. J Cell Biochem. 1995; 59:443-52.

76. Schiestl RH, Gietz RD. High efficiency transformation of intact yeast cells using single stranded nucleic acids as a carrier. Curr Genet. 1989:16:339-46.

77. Frøkjaer-Jensen C, Davis MW, Hopkins CE, Newman BJ, Thummel JM, Olesen S-P, et al. Single-copy insertion of transgenes in Caenorhabditis elegans. Nat Genet. 2008:40:1375-83. 
78. Katoh K, Standley DM. MAFFT multiple sequence alignment software version 7: improvements in performance and usability. Mol Biol Evol. 2013; 30:772-80.

79. Price MN, Dehal PS, Arkin AP. FastTree 2-approximately maximumlikelihood trees for large alignments. PLoS One. 2010;5, e9490.

80. Letunic I, Bork P. Interactive Tree Of Life v2: online annotation and display of phylogenetic trees made easy. Nucleic Acids Res. 2011;39:W475-8.

81. Stothard P. The sequence manipulation suite: JavaScript programs for analyzing and formatting protein and DNA sequences. Biotechniques. 2000; 28:1102-4.

82. Chen B, Gilbert LA, Cimini BA, Schnitzbauer J, Zhang W, Li G-W, et al. Dynamic imaging of genomic loci in living human cells by an optimized CRISPR/Cas system. Cell. 2013;155:1479-91.

83. Farboud B, Meyer BJ. Dramatic enhancement of genome editing by CRISPR/ Cas9 through improved guide RNA design. Genetics. 2015;199:959-71.

84. Dickinson DJ, Pani AM, Heppert JK, Higgins CD. Goldstein B. streamlined genome engineering with a self-excising drug selection cassette. Genetics. 2015;200:1035-49.

85. Friedland AE, Tzur YB, Esvelt KM, Colaiácovo MP, Church GM, Calarco JA. Heritable genome editing in C. elegans via a CRISPR-Cas9 system. Nat Methods. 2013;10:741-3.

86. Kapitein LC, Yau KW, Hoogenraad CC. Microtubule dynamics in dendritic spines. Methods Cell Biol. 2010;97:111-32.

87. Vizcaíno JA, Deutsch EW, Wang R, Csordas A, Reisinger F, Ríos D, et al. ProteomeXchange provides globally coordinated proteomics data submission and dissemination. Nat Biotechnol. 2014;32:223-6.

Submit your next manuscript to BioMed Central and we will help you at every step:

- We accept pre-submission inquiries

- Our selector tool helps you to find the most relevant journal

- We provide round the clock customer support

- Convenient online submission

- Thorough peer review

- Inclusion in PubMed and all major indexing services

- Maximum visibility for your research

Submit your manuscript at www.biomedcentral.com/submit
Biomed Central 